\title{
Comprehensive analysis of the Gossypium hirsutum L. respiratory burst oxidase homolog (Ghrboh) gene family
}

Wei Wang, Dongdong Chen, Dan Liu, Yingying Cheng, Xiaopei Zhang, Lirong Song, Mengjiao Hu, Jie Dong and Fafu Shen ${ }^{*}$ (D)

\begin{abstract}
Background: Plant NADPH oxidase (NOX), also known as respiratory burst oxidase homolog (rboh), encoded by the rboh gene, is a key enzyme in the reactive oxygen species (ROS) metabolic network. It catalyzes the formation of the superoxide anion $\left(\mathrm{O}_{2}{ }^{--}\right)$, a type of ROS. In recent years, various studies had shown that members of the plant rboh gene family were involved in plant growth and developmental processes as well as in biotic and abiotic stress responses, but little is known about its functional role in upland cotton.

Results: In the present study, 26 putative Ghrboh genes were identified and characterized. They were phylogenetically classified into six subfamilies and distributed at different densities across 18 of the 26 chromosomes or scaffolds. Their exon-intron structures, conserved domains, synteny and collinearity, gene family evolution, regulation mediated by cisacting elements and microRNAs (miRNAs) were predicted and analyzed. Additionally, expression profiles of Ghrboh gene family were analyzed in different tissues/organs and at different developmental stages and under different abiotic stresses, using RNA-Seq data and real-time PCR. These profiling studies indicated that the Ghrboh genes exhibited temporal and spatial specificity with respect to expression, and might play important roles in cotton development and in stress tolerance through modulating NOX-dependent ROS induction and other signaling pathways.

Conclusions: This comprehensive analysis of the characteristics of the Ghrboh gene family determined features such as sequence, synteny and collinearity, phylogenetic and evolutionary relationship, expression patterns, and cis-elementand miRNA-mediated regulation of gene expression. Our results will provide valuable information to help with further gene cloning, evolutionary analysis, and biological function analysis of cotton rbohs.
\end{abstract}

Keywords: Rboh, Reactive oxygen species, Upland cotton, Expression patterns, Gene family

\section{Background}

Plants are continually exposed to biotic and abiotic stresses, which negatively affect their growth and yield, causing enormous losses in agriculture worldwide. These stressors, such as pathogenic infections, drought, extreme temperatures and salt, lead to the over-accumulation of reactive oxygen species (ROS). ROS, including the superoxide anion $\left(\mathrm{O}_{2}{ }^{--}\right)$, hydroxyl radical $(\cdot \mathrm{OH})$, hydrogen peroxide $\left(\mathrm{H}_{2} \mathrm{O}_{2}\right)$, singlet oxygen $\left({ }^{1} \mathrm{O}_{2}\right)$, ozone $\left(\mathrm{O}_{3}\right)$ and nitric oxide (NO), have long been known to act as signal

\footnotetext{
* Correspondence: cotton1@sdau.edu.cn

State Key Laboratory of Crop Biology, College of Agronomy, Shandong Agricultural University, NO. 61 Daizong Street, Tai'an, Shandong 271018, People's Republic of China
}

molecules in plants, regulating growth and development [1], programmed cell death (PCD) [2], hormone signaling [3], and responses to biotic and abiotic stresses [4, 5]. Excessive accumulation of ROS causes membrane damage, protein oxidation and DNA lesions, and can even lead to irreparable metabolic dysfunctions and cell death [6].

Plasma membrane NADPH oxidase (NOX) is a key enzyme involved in ROS formation. Plant NOX, known as respiratory burst oxidase homolog (rboh) and encoded by rboh genes, is a homolog of the mammalian NOX catalytic subunit known as gp91 ${ }^{\text {phox }}$ [7]. The available crystal structures of classical plant rboh proteins have revealed the presence of two $\mathrm{Ca}^{2+}$-binding EF-hand motifs, six transmembrane domains and FAD- and NADPH-binding domains from the 
$\mathrm{N}$-terminal region to the C-terminal region [8]. The plant rboh gene comprises a multiple gene family. In plants, OsrbohA was the first rboh gene identified in rice (Oryza sativa L.) [9], and subsequent studies indicated that different rboh genes in lower plants, monocots and dicots constituted a multigene family [10]. As more and more plant genomes are available, the rboh gene family has been characterized in some plant species, such as Arabidopsis thaliana (L.) Heynh [11], O. sativa L. [12], Hordeum vulgare L. [13], Medicago truncatula Gaertn. [14], Vitis vinifera L. [15], Malus domestica Mill. [16] and Hevea brasiliensis Muell. Arg. [17]. The genome of $A$. thaliana contains ten Atrboh genes, and it has been shown, by a meta-analysis of Genevestigator microarray datasets, that AtrbohD is the most highly expressed gene, whereas AtrbohE and AtrbohH show their highest expression in mature siliques, with very low expression in leaf tissues [12]. Expression of the Atrboh gene family is also induced in response to hormonal treatments and abiotic stresses. AtrbohB and AtrbohE show contrasting expression in response to the hormones abscisic acid (ABA), auxin and ethylene [12]. With the exception of heat stress conditions, under which all Atrbohs are found to be down-regulated, other abiotic stress conditions (drought, osmotic, salt, heat, cold, wounding, hypoxic and genotoxic) involve a mixture of upand down-regulation of various Atrbohs [12, 18, 19]. In addition, the Atrboh gene family is also involved in regulating growth and development [1], and programmed cell death [2]. There are 9, 7 and 9 rboh genes in the genomes of rice, grape and apple, respectively, and the genome-wide analyses of $r b o h$ gene family in these plants reveal that the expression patterns of rboh genes varied under different treatments, indicating diverse functions in plant stress responses.

Allotetraploid upland cotton (Gossypium hirsutum L.) is both the world's most important fiber crop as well as a source of seed oil and protein meal, and a model polyploid crop [20]. In a previous study, inhibiting the activity of the NADPH oxidase with diphenyleneiodonium (DPI) caused inhibition of both ROS formation and fiber cell elongation, a finding which reveals that NADPH oxidase is crucial for cotton fiber development [21]. However, a comprehensive characterization analysis of upland cotton rboh genes has not yet been reported, and no rboh gene of upland cotton has even been cloned. As cotton genomics develops, the release of the upland cotton genome sequence now allows a comprehensive genome-scale identification and analysis of Ghrboh genes [22-25]. In this study, we performed a genomescale analysis of the rboh gene family in the upland cotton genome. Detailed information on genomic organization, gene structure, phylogenetic relationships and synteny with the diploid cotton rboh gene families were also reported. Furthermore, cis-elements in the putative promoters and microRNA (miRNA) target sites of Ghrbohs were analyzed, and the expression profiles of members of the Ghrboh gene family were investigated using RNA-Seq data and were analyzed using qPCR.

\section{Results \\ Identification of $R$ boh genes in the upland cotton genome}

To identify all the rboh genes in the upland cotton genome, HMMER and BLAST searches were performed using ten rboh genes from $A$. thaliana and conserved domains of rboh proteins as the queries. A total of 26 putative Ghrboh genes were identified. The distribution and density of Ghrboh genes on chromosomes (scaffolds) was not uniform. 18 chromosomes (scaffolds) carried Ghrboh genes, with 12 (chromosomes A1, A3, A8, A11, A12, D1, D8, D11, scaffold413_A2, scaffold3396_ A12, scaffold3404_A12 and scaffold4588_D12) each carrying 1 Ghrboh gene and 4 (chromosomes A5, D3, D5 and D12) possessing 2 Ghrboh genes each, while the other 2 (chromosomes A7 and D7) involved each contained 3 Ghrboh genes. Additionally, half of the 26 Ghrboh genes were evenly distributed among Dt chromosomes (from tetraploid D) and At chromosomes (from tetraploid A). According to their localization in the G. hirsutum genome, we named these genes Ghrboh1-26, and the gene names, sequence IDs and genomic positions are shown in Table 1.

\section{Sequence analysis and functional annotation}

The result of Ghrboh gene structure analysis revealed that the numbers of exons in each gene varied between 10 and 15, with the lowest numbers of exons being in Ghrboh2 and Ghrboh7, and the highest number in Ghrboh17. The genes clustering into the same group showed similar gene structures (Fig. 1a and b). Among the upland cotton rboh gene family, the order and approximate sizes of the exons were relatively conserved, compared with the more variable size of the introns (Fig. $1 b)$. For instance, the spacing between the third and fourth exon of Ghrboh17, as well as between the fourth and fifth exon, was particularly variable, as seen in the corresponding exons of Ghrboh5, Ghrboh9, Ghrboh13, Ghrboh23 and Ghrboh24. The results were consistent with those previously reported in Arabidopsis, barley, rice and grape $[12,13,15]$.

The physico-chemical analysis of the predicted Ghrboh proteins encoded by candidate Ghrboh genes showed that the lengths, molecular masses, isoelectric points and instability indices of rboh proteins were within the ranges of 721-940 amino acids (aa), 81.22$107.08 \mathrm{kDa}, 8.65-9.63$ and $36.86-50.56$, respectively (Table 1). All the predicted upland cotton rboh protein were alkaline. Other than Ghrboh5, Ghrboh6, Ghrboh9, Ghrboh10 and Ghrboh13, most predicted Ghrboh proteins were unstable (Table 1). Computational prediction 


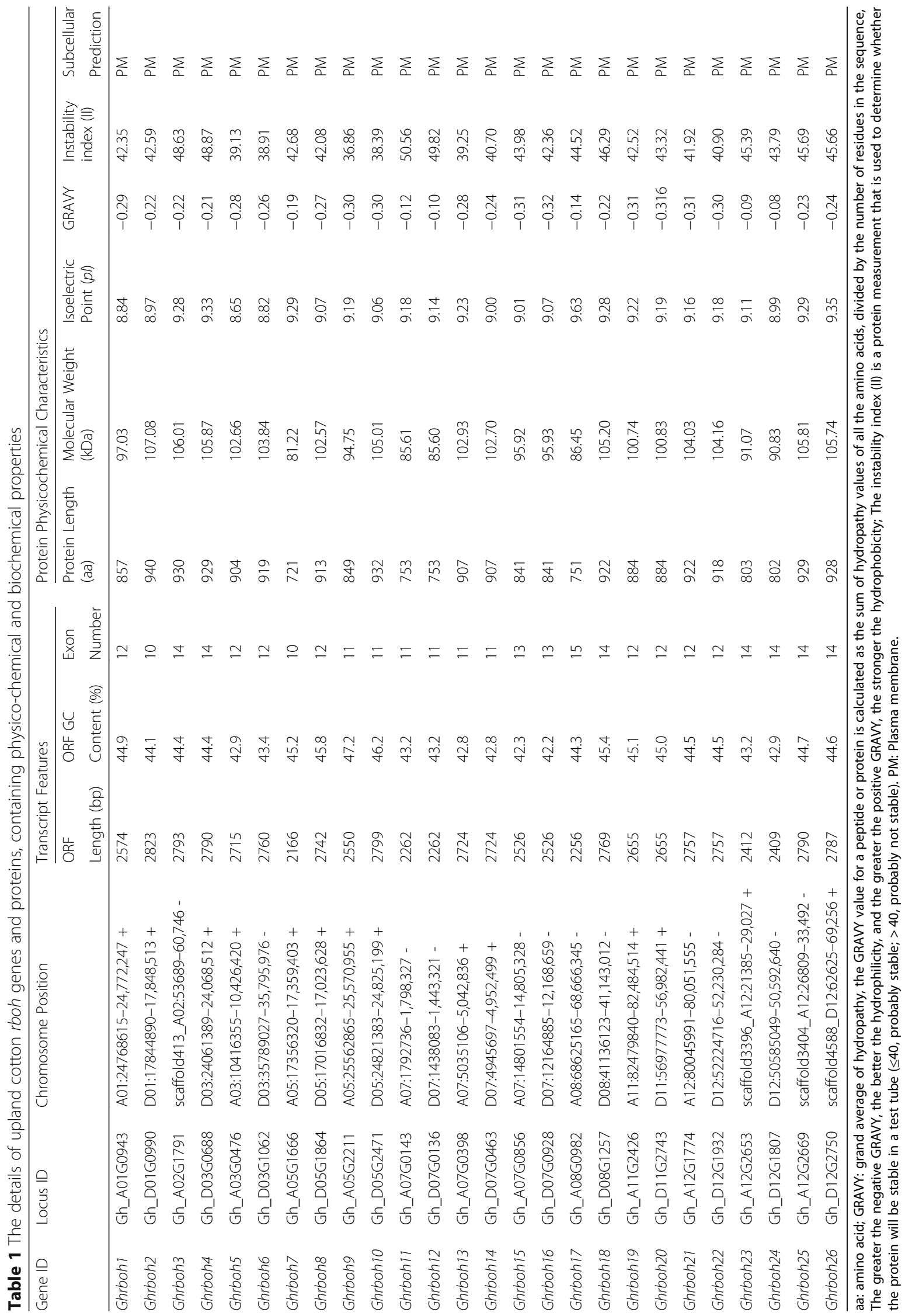



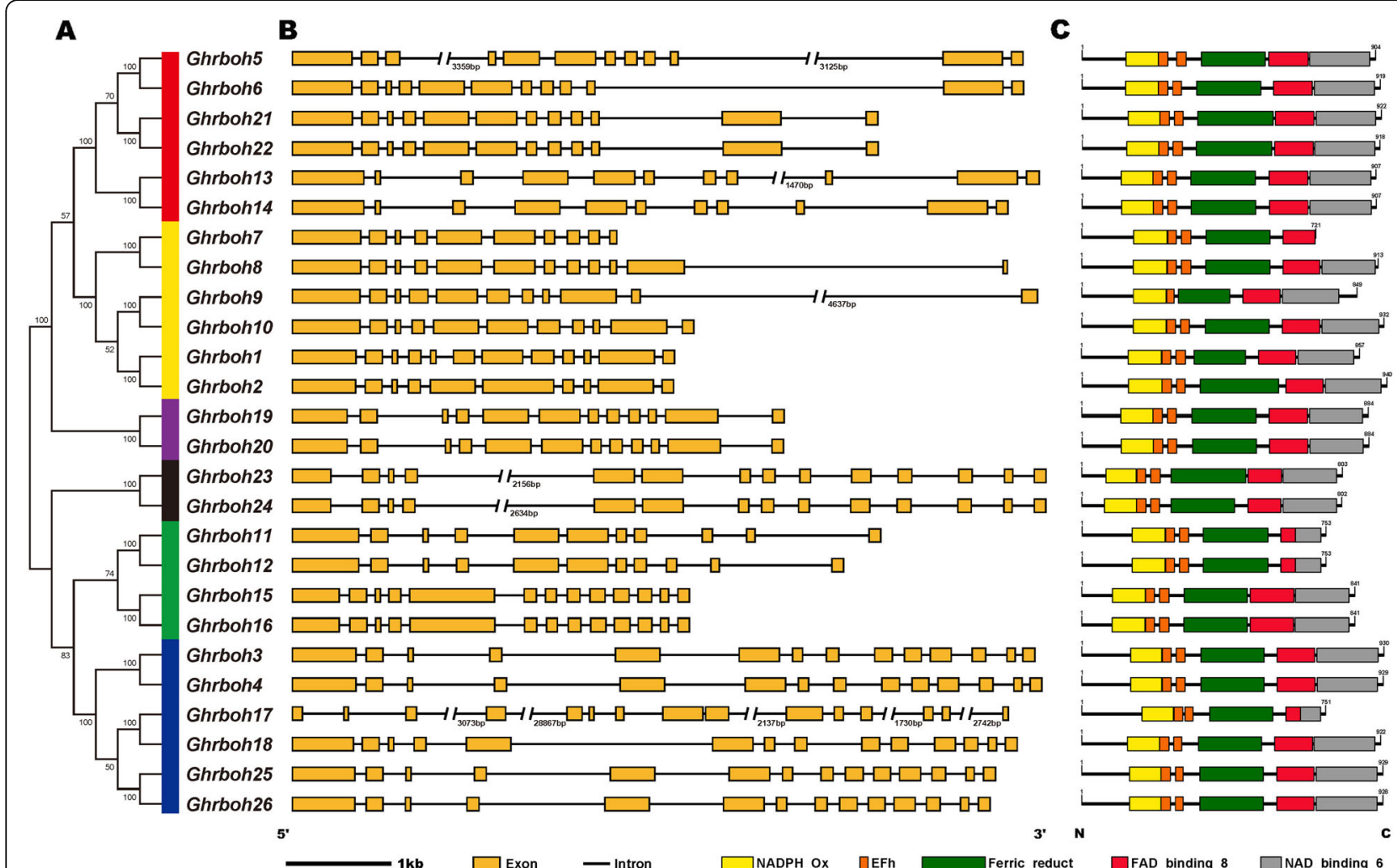

Fig. 1 Cluster analysis, gene structure and domain analysis of upland cotton rboh gene family. (A) Phylogenetic tree of G. hirsutum rbohs constructed with MEGA 6.0 by the NJ method. Bootstrap values from 1000 replicates are indicated at each branch. Group I to VI represented by red, yellow, purple, black, green, and blue, respectively. (B) Exon-intron structures of Ghrboh genes. Yellow boxes and black horizontal lines indicated exons and introns, respectively. (C) Domain compositions of upland cotton rbohs. Only major domains were presented here based on our database searches in Pfam database (http://pfam.xfam.org/)

of protein localization indicated that all Ghrboh proteins were localized in the plasma membrane. The information in the literature indicated that Ghrboh proteins were localized to the plasma membrane and transferred electrons from cytosolic $\mathrm{NAD}(\mathrm{P}) \mathrm{H}$ to an electron acceptor and catalyzed the formation of apoplastic $\mathrm{O}_{2}{ }^{-}$ [9]. This corroborated our findings.

The conserved domains of candidate Ghrboh protein sequences were analyzed (Table 1). Although the Ghrboh proteins were of different sizes, their major functional domains were similar. Based on the domain analysis, all 26 predicted Ghrboh proteins contained one NADPH_Ox domain (PF08414), two elongation factor (EF)-hand motifs (PF00036), one Ferri_reduct domain (PF01794), one FAD-binding_8 domain (PF08022) and one NAD-binding 6 domain (PF08030) from $\mathrm{N}$-terminus to $\mathrm{C}$-terminus, except for Ghrboh9, which contained only one EF-hand motifs (Fig. 1c).

\section{Synteny and collinearity analysis}

To analyze the synteny and collinearity relationships of cotton rboh genes, we identified the orthologous and paralogous genes among G. hirsutum, G. raimondii and G. arboreum (Fig. 2, Additional file 1: Table S1 and Additional file 1: Table S2). From a Gossypium evolutionary point of view, we can deem that one rboh gene in the diploid species G. raimondii corresponds to 1 homologous gene in $G$. arboreum and 2 homologs, one each from the At and Dt subgenomes, in tetraploid G. hirsutum. We found that, of all 26 rboh genes identified in the G. hirsutum genome, 22 Ghrbohs had orthologs in G. raimondii and G. arboreum, with 10 showing an A genome origin and $12 \mathrm{D}$ genome origin. Of 14 Garboh genes, 13 had orthologs in G. raimondii (Fig. 2 and Additional file 1: Table S1). The results indicated that the A- and D-subgenomes evolved independently after polyploid formation.

We further identified gene losses in syntenic blocks, among the cotton rboh genes that had no orthologs. Ghrboh3/23 and Ghrboh8 had no orthologs in G. raimondii and G. arboreum, respectively, Garboh2/12/13/ 14 had no orthologs in G. hirsutum, Grrboh4 had no orthologs in G. hirsutum, and Garboh14 had no orthologs in G. hirsutum or G. raimondii. Considering the evolutionary history of cotton $[24,25]$, we hypothesized that the orthologous gene of Garboh14 in G. raimondii was lost during divergence between G. raimondii and G. arboreum from their common ancestor (approximately 


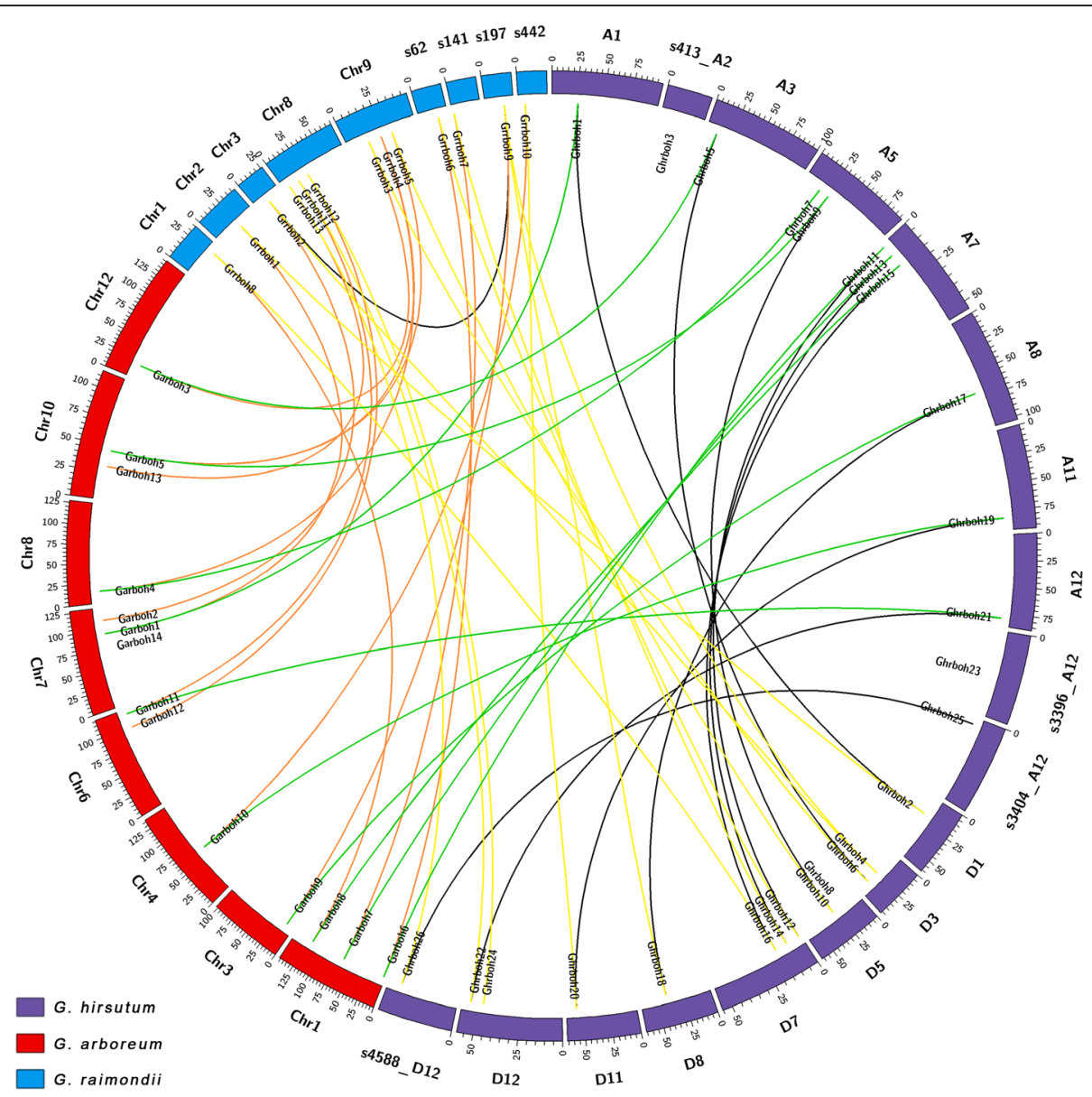

Fig. 2 Chromosomal location and synteny relationships of rboh genes from G. hirsutum, G. raimondii and G. arboretum. G. hirsutum, G. raimondii and $G$. arboretum chromosomes are indicated in purple, blue and red, respectively. The putative orthologous rboh genes between $G$. hirsutum and G. raimondii, G. hirsutum and G. arboretum, and G. raimondii and G. arboretum are connected by yellow, red and orange lines, respectively. Black lines connect the putative paralogous genes. s413_A2, s, scaffold

2 13 million years ago, MYA), and the orthologs of Garboh2/12/13 in G. hirsutum were lost when the allotetraploid was formed approximately 1 1.5 MYA. These results indicated that more genes were lost from the At subgenome than from the Dt subgenome during the formation of G. hirsutum, which was consistent with the findings of a previous study [22]. Apart from gene loss, the result might also be artefacts, resulting from the sequencing methods used and genome assembly quality in different cotton species, or from errors of assembly and annotation in partial chromosomal regions. This possibility needs further investigation.

Gene duplications, occurring during the course of cotton evolution, have led to the development of new gene functions [26]. Genes might be duplicated by mechanisms other than whole-genome duplication (WGD), such as tandem, proximal and/or dispersed duplications, each of which might make different contributions to evolution [27]. To analyze the relationship between cotton rboh genes and gene duplication events, we characterized ten pairs of paralogous genes in the G. hirsutum genome, and one pair in the G. raimondii genome (Fig. 2 and Additional file 1: Table S2) and classified the duplicate genes. The duplicate genes of the Ghrboh gene family could be classified into WGD/segmental or dispersed duplicates. With the exception of Ghrboh3/8/23, which were dispersed duplicates, the rest of the Ghrboh genes were WGD/segmental duplicates, with tandem duplications not being observed. WGD/segmental duplicates were inferred by the presence of anchor genes in collinear blocks, whereas dispersed duplicates were paralogs that were neither near one another on chromosomes, nor did they show conserved synteny. These results indicated that WGD/segmental duplications mainly contributed to the expansion of the Ghrboh gene family in upland cotton.

\section{Phylogenetic and evolutionary analysis}

To investigate the evolutionary relationships between rboh proteins among upland cotton and other Gossypium spp., 2 phylogenetic trees were independently constructed using 
predicted full-length amino acid sequences and the MEGA 6.0 software with the neighbor-joining (NJ) method (Fig. 3 and Fig. 1a). The rboh genes of 3 cotton species were clustered into 6 groups, which showed accordance with previous phylogenetic analyses of plant rbohs $[15,28]$. Groups I to VI are represented by red, yellow, purple, black, green, and blue, respectively (Fig. 1a).

Using the same method as used to identify rboh genes in the upland cotton genome, we also searched for rboh genes in the genomes of lower aquatic to higher terrestrial plants. Among green alga, four Crrbohs were identified from Chlamydomonas reinhardtii P.A. Dangeard, but there were no rboh genes in the genome of the other green alga investigated, namely Micromonas pusilla (R. W. Butcher) I. Manton \& M. Parke, Ostreococcus lucimarinus and Volvox carteri F.Stein. 4 Pprbohs were identified from the moss, Physcomitrella patens (Hedw.) Bruch \& Schimp. In the spikemoss, Selaginella moellendorffii Hieron., a member of the Pteridophyta, there were 10 Smrboh genes. In the genome of the understory shrub Amborella trichopoda Baill., 6 Amtrbohs were identified.
Among monocots, the number of rbohs was 7 in Ananas comosus (L.) Merr., 9 each in Brachypodium distachyon (L.) P.Beauv. and O. sativa L., and 10 each in Sorghum bicolor (L.) Moench and Musa acuminata Colla. Among eudicots, the number of rbohs was 7 in each of Theobroma cacao L., Medicago truncatula Gaertn. and V. vinifera L., 10 each in A. thaliana, Malus domestica Borkh. and Daucus carota L., 13 in G. raimondii, and 14 in G. arboretum (Additional file 1: Table S3). Evolutionary analysis using 20 species from lower aquatic to higher terrestrial plants showed that rboh genes first appeared in the green algae (C. reinhardtii) and the number of genes increased dramatically in pteridophytes $(S$. moellendorffii), then stayed relatively stable until the upland cotton evolved (Additional file 1: Figure S1). This finding was consistent with a WGD event resulting in tetraploid cotton after two diploid cotton species reunited geographically around 1 2 MYA [29].

In terms of Gossypium rbohs, the total number in $G$. raimondii and $G$. arboretum, which were considered to be the A-genome ancestor and D-genome ancestor,

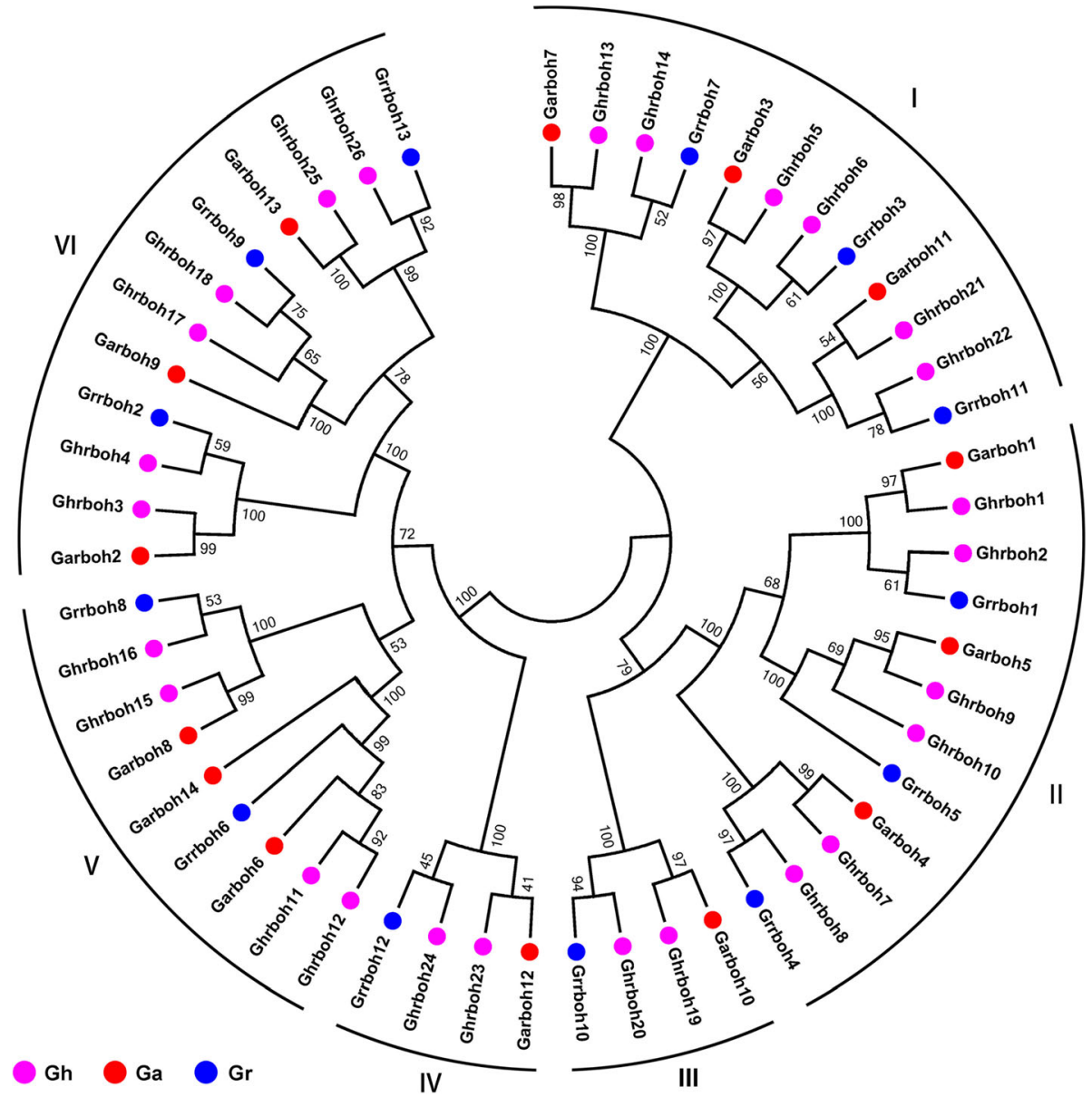

Fig. 3 Neighbor-joining (NJ) phylogenetic tree of the rboh gene family among Gossypium. The tree was constructed with predicted full length rboh amino acid sequences from in G. hirsutum (Gh), G. arboreum (Ga), and G. raimondii (Gr) 
respectively, of G. hirsutum, was 27, which was nearly equal to that in G. hirsutum. All other upland cotton rboh genes were clustered together as either G. raimondii or G. arboretum rboh genes. This finding was consistent with the hypothetical origins and history of allotetraploid cotton [29].

In addition, to calculate the evolutionary time of Ghrboh genes and gain more insights into the divergence of the upland cotton rboh gene family after polyploidization, an estimation of their non-synonymous $(\mathrm{Ka})$ and synonymous $(K s)$ nucleotide substitutions and their ratio $(K a / K s)$ during evolution were calculated using the add_ka_and ks_to_collinearity.pl program of MCScanX software (Additional file 1: Table S2). The $K a / K s$ ratio is a measure used to examine the mechanisms of gene duplication evolution after divergence from an ancestor and to estimate the balance between neutral selection $(K a / K s=1)$, purifying selection $(K a / K s<1)$ and positive selection $(K a / K s>1)$ [30]. The analysis demonstrated that nine of the ten Ghrboh paralogous pairs had $K a / K s$ ratios less than 1, indicating that the Ghrboh gene family had been influenced principally by high purifying selection, while one pair of duplicated genes had a $K a / K s$ ratio greater than 1, implying that they had evolved under positive selection (Additional file 1: Table S2). This result revealed that the Ghrboh genes were evolving slowly and had conserved characteristics at the protein level. According to the neutral substitution $(r)$ rate of $2.6 \times 10^{-9}$ synonymous mutations per locus per year, the estimated divergence time $(t)$ was calculated from the equation " $t=K s / 2 r$ " MYA [31]. The ten paralogous pairs were calculated to have diverged between 3.32 MYA ( $K s=0.0173)$ and 16.88 MYA $(K s=0.0878)$, with an average of 8.34 MYA (Additional file 1: Table S2). These results suggested that the expansion of Ghrboh genes in upland cotton mostly arose as a result of WGD/ segmental events during the divergence of one common ancestor into G. raimondii and G. arboreum approximately 2 13 MYA [22].

\section{Expression profiles of Ghrboh genes in different tissues/ organs and development stages}

Gene expression profiles are closely associated with gene functions. Plant rboh genes are involved in growth and development [1], programmed cell death [2] and so on. To preliminarily study their biological functions in upland cotton with respect to different developmental processes, we initially collected the transcript profiles from root, stem, leaf, petal, torus, stamen, pistil, calycle, and ovules at $-1 / 0$ / $1 / 3 / 5 / 10 / 20 / 25$ days post anthesis (dpa) and fibers at $5 / 10$ / 20/25 dpa from RNA-Seq data published by Zhang et al. using the G. hirsutum cultivar TM-1 [24] (Fig. 4).

Generally, the candidate Ghrboh genes showed very dynamic expression profiles in the afore-mentioned eight tissues and/or organs. Of the 26 candidate genes, six Ghrboh genes (Ghrboh6/9/10/21/22/26) were highly expressed in most of the eight tissues and/or organs, whereas the expression of a further 6 Ghrboh genes (Ghrboh3/4/13/14/19/25) were higher in some tissues and/or organs, but much lower or even barely detectable in others (Fig. 4a). For instance, the expression of Ghrboh25 was higher in stem and torus, lower in leaf, and almost undetectable in the root, petal, stamen, pistil, and calycle. Furthermore, 12 Ghrboh genes (Ghrboh1/2/ $5 / 7 / 8 / 11 / 12 / 17 / 18 / 20 / 23 / 24)$ were expressed at very low levels or were even barely detectable in all eight tissues and/or organs tested. Remarkably, Ghrboh15 and Ghrboh16 were expressed constitutively in the stamen,

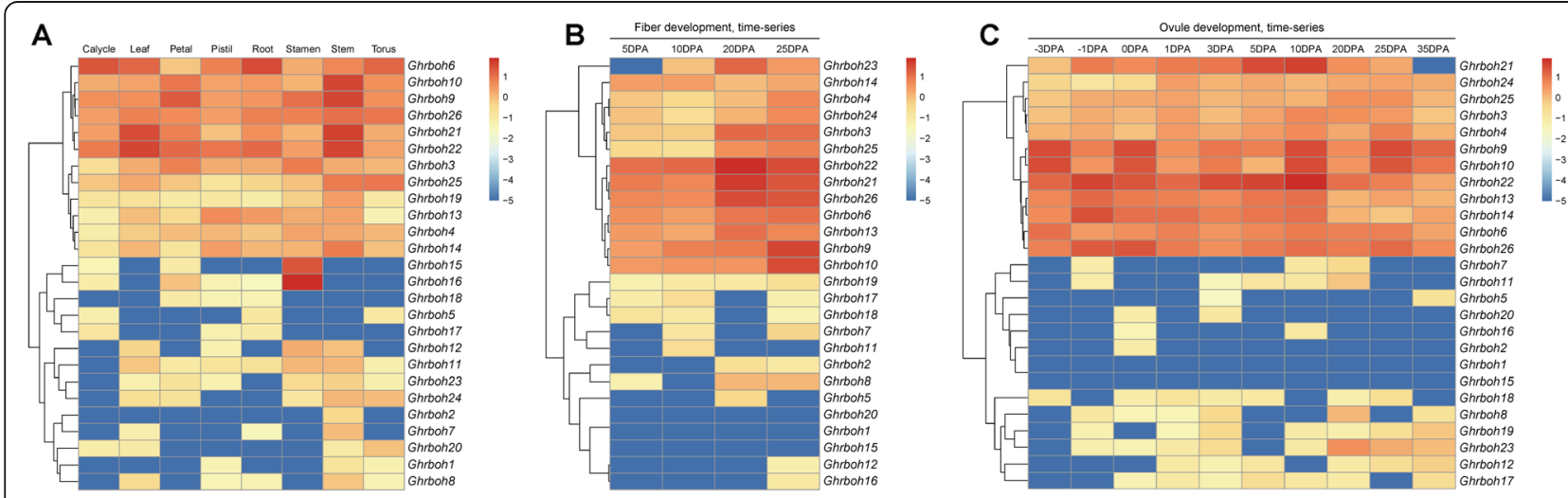

Fig. 4 Expression profiles of Ghrbohs in different tissues/organs and development stages. The $\log _{2}$ of FPKMs values calculated by RNA-Seq data were shown as a heat map. The colors of the bar shown to the right of the heat-map varied from red to blue representing the relative expression levels from high to low. FPKMs data was obtained from ccNET (http://structuralbiology.cau.edu.cn/gossypium/) and CottonFGD (https://cottonfgd.org/). (A) The heat-map showed the hierarchical clustering of the relative expression of 26 Ghrbohs in root, stem, leaf, petal, torus, stamen, pistil, calycle. (B) The heat-map showed the hierarchical clustering of the relative expression of 26 Ghrbohs in fibers at 5, 10, 20 and $25 \mathrm{dpa}$. (C) The heat-map showed the hierarchical clustering of the relative expression of 26 Ghrbohs in ovules at $-3,-1,0,1,3,5,10,20,25$ and $35 \mathrm{dpa}$ 
but their expression levels in other tissues and/or organs were very low (Fig. 4a).

In addition to the tissue- or organ-specific expression profiles of Ghrbohs, we also analyzed the expression of all candidate Ghrboh genes during the cotton fiber and ovule development processes, using microarray expression data (Fig. 4b and Fig. 4c). The results showed that not all the candidate Ghrboh genes were expressed at the different developmental stages of upland cotton fibers and ovules. Overall, there were two types of fiber/ovule gene expression profiles: those which were expressed more-or-less constitutively, and those which were expressed at extremely low or even undetectable levels during the process of fiber and ovule development. Another interesting scenario was that, during fiber development, Ghrboh1/15/20 did not show any detectable expression, whereas three Ghrboh genes (Ghrboh21/22/26) showed expression levels at $20 \mathrm{dpa}$ between 2- and 6-fold higher than those observed at other stages, while Ghrboh9/10 exhibited continuous increases in expression throughout fiber development (Fig. 4b). During ovule development, Ghrboh1/15 did not show any detectable expression, Ghrboh21 and Ghrboh 22 were down-regulated at the stage from $10 \mathrm{dpa}$ to $20 \mathrm{dpa}$, Ghrboh9 was down-regulated at the stage from $0 \mathrm{dpa}$ to $1 \mathrm{dpa}$ and from $10 \mathrm{dpa}$ to $20 \mathrm{dpa}$, and Ghrboh10 was up-regulated at the stage from $5 \mathrm{dpa}$ to $10 \mathrm{dpa}$ and down-regulated at the stage from 10 dpa to 20 dpa (Fig. 4c).

Since NADPH oxidase is crucial for cotton fiber development [21], gene expression patterns in fibers and ovules at different time points after flowering were studied using real-time quantitative (qPCR) (Additional file 1: Figure S2 and Additional file 1: Figure S3). We used the transcript levels of the Ghrboh genes in the young leaves (YL) as references and set the reference value to 1 . The qPCR analysis revealed that all of the 26 Ghrboh genes were differentially expressed in each of the developmental stages of fiber (5, $10,20$ and $25 \mathrm{dpa})$ and ovule $(-1,0,3,5,10,20$ and 25 dpa) tested in upland cotton. During fiber development, 4 Ghrboh genes (Ghrboh10/14/25/26) were significantly differentially expressed at $5 \mathrm{dpa}, 3$ Ghrboh genes (Ghrboh3/ $18 / 20$ ) were significantly differentially expressed at $10 \mathrm{dpa}$, 3 Ghrboh genes (Ghrboh4/9/17) were significantly differentially expressed at 5 and $10 \mathrm{dpa}, 3$ Ghrboh genes (Ghrboh8/ 12/15) were significantly differentially expressed at 10 and 20 dpa, Ghrboh13 and Ghrboh22 were significantly differentially expressed at 5 and $20 \mathrm{dpa}$, Ghrboh24 was significantly differentially expressed at 5 and $25 \mathrm{dpa}$, and Ghrboh 21 was significantly differentially expressed at 5, 10 and $20 \mathrm{dpa}$ (Additional file 1: Figure S2).

During ovule development, 6 Ghrboh genes (Ghrboh2/ $3 / 4 / 8 / 17 / 19)$ were significantly differentially expressed at -1 and 0 dpa, 5 Ghrboh genes (Ghrboh7/11/15/16/18) were significantly differentially expressed at $0 \mathrm{dpa}, 3$
Ghrboh genes (Ghrboh13/14/21) were significantly differentially expressed at 0, 5 and $10 \mathrm{dpa}$, Ghrboh12 was significantly differentially expressed at -1 and $5 \mathrm{dpa}$, Ghrboh23 was significantly differentially expressed at 0 and $25 \mathrm{dpa}$, Ghrboh22 was significantly differentially expressed at 3 and $5 \mathrm{dpa}$, and Ghrboh 24 was significantly differentially expressed at 5 and $10 \mathrm{dpa}$ (Additional file 1: Figure S3). These qPCR results will lay the groundwork for further cloning and functional analysis of the Ghrboh gene family.

All these results indicated that expression of the members of the Ghrboh gene family exhibited temporal and spatial specificity and might be involved in the growth and development of different tissues or organs of upland cotton.

\section{Expression profiles of Ghrboh genes under different abiotic stress treatments}

Previous studies had revealed that plant rboh genes were widely associated with abiotic stress responses under normal and stressed growth conditions $[4,11]$. To determine whether the Ghrboh genes responded to stress conditions, we examined the expression profiles of all 26 predicted Ghrboh genes in response to a series of abiotic stresses (hot, cold, drought (polyethylene glycol, PEG) and salt), using RNA-Seq data (Fig. 5) and qPCR (Additional file 1: Figure S4). As shown in Fig. 5, the upland cotton rboh gene family was differentially expressed in the leaves under hot, cold, drought and salt stress conditions. Under high-temperature stress treatment, a total of 9 genes (Ghrboh3/4/6/9/10/21/22/25/26) showed continuous and stable expression, whereas Ghrboh2/15/16/ $17 / 23$ did not show any detectable expression, suggesting that they were not involved in heat-stress response. In addition, Ghrboh1/5/13/14 were down-regulated at the $1 \mathrm{~h}$ time point of the heat stress treatment (Fig. 5a). Under cold stress treatment, a total of 11 genes (Ghrboh3/4/5/6/9/10/14/21/22/25/26) showed continuous and stable expression, whereas expression of Ghrboh17/20/23 was not induced by cold treatment, and a total of 7 genes were down-regulated at early time points and up-regulated after experiencing a longer cold treatment period (Fig. 5b). Under PEG treatment, a total of 12 genes were expressed continuously and stably, among which Ghrboh6/9/10/21/22/25/26 showed higher expression, and Ghrboh3/4/14/18/24 showed lower expression. Ghrboh2/8/12/15/17 were not induced by PEG treatment, and a total of 5 genes were down-regulated at early treatment time points and up-regulated after experiencing a longer PEG treatment (Fig. 5c). Under salt treatment, a total of eleven genes were expressed continuously and stably, among which Ghrboh6/9/10/21/22/ 25/26 exhibited higher expression, and Ghrboh3/4/5/14 showed lower expression. Ghrboh11/16/23 were not 

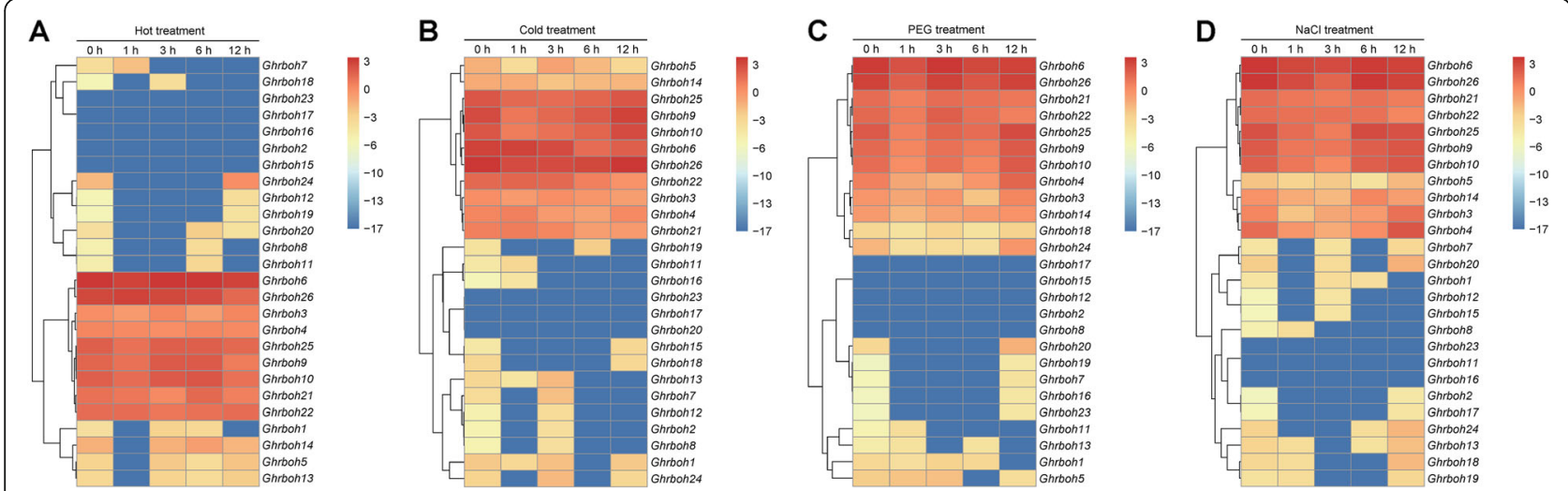

Fig. 5 Expression profiles of Ghrbohs under different stress treatments. The $\log _{2}$ of FPKMs values calculated by RNA-Seq data were shown as a heat map. The colors of the bar shown to the right of the heat-map varied from red to blue representing the relative expression levels from high to low. FPKMs data was obtained from ccNET (http://structuralbiology.cau.edu.cn/gossypium/) and CottonFGD (https://cottonfgd.org/). The heatmap showed the hierarchical clustering of the relative expression of Ghrbohs under hot, cold, PEG and salt treatments (A-D)

induced by salt treatment, and Ghrboh1/2/7/8/12/15/17/ 20 were expressed at extremely low or even undetectable levels during salt treatment (Fig. 5d).

To determine gene expression under abiotic stress, expression in leaf tissue from plants exposed to salinity or drought conditions was determined using qPCR. The results showed that the expression patterns of Ghrbohs were complex under salinity or drought treatments. Under salinity stress, 4 Ghrboh genes (Ghrboh9/14/21/22) were upregulated and maintained a relatively high expression level, with Ghrboh14 and Ghrboh22 exhibited approximately 3- to 30-fold induction. In contrast, expression of 3 genes (Ghrboh4/15/24) was down-regulated in response to salinity stress. 3 genes (Ghrboh3/10/23) were upregulated at $6 \mathrm{~h}, 3$ genes (Ghrboh10/13/20) were upregulated at $12 \mathrm{~h}, 5$ genes (Ghrboh2/8/11/16/17) were upregulated at $6 \mathrm{~h}$ and $12 \mathrm{~h}$. Ghrboh18 and Ghrboh19 were significantly down-regulated at $3 \mathrm{~h}$, and significantly upregulated over the rest of the $\mathrm{NaCl}$ treatment. The results suggested that these genes, acting as positive or negative regulators, were involved in the response of upland cotton to salinity stress (Additional file 1: Figure S4).

Under drought stress, 12 genes (Ghrboh2/5/6/8/9/10/ $11 / 16 / 19 / 21 / 22 / 23)$ were down-regulated in response to drought stress. In contrast, Ghrboh3 and Ghrboh7 were up-regulated and exhibited approximately 2- to 20-fold induction. In addition, 5 genes (Ghrboh1/14/17/18/24) were significantly up-regulated at $3 \mathrm{~h}, 2$ genes (Ghrboh25/26) were significantly up-regulated at $1 \mathrm{~h}$ and $3 \mathrm{~h}$, Ghrboh4 was significantly up-regulated at $6 \mathrm{~h}$, Ghrboh15 was significantly up-regulated at $1 \mathrm{~h}$ and $12 \mathrm{~h}$ and significantly down-regulated or exhibited no significant expression differences over the rest of the PEG treatment (Additional file 1: Figure S4).

The reasons underlying the comprehensive expression profiles of these genes might indicate their vital functions in response to heat, cold, drought or salt treatment. The results of the expression analysis suggested that the rboh gene family of upland cotton may be important in terms of stress responses as well as developmental processes.

\section{Cis-element analysis of putative Ghrboh promoters}

We regarded the 1.5-kb genomic sequences upstream from the transcription start site (TSS) of each upland cotton rboh gene as putative promoter regions and used the PlantCARE tool to identify the presence of cis-elements which could be controlling the expression of the Ghrboh genes. All 26 putative Ghrboh promoters possessed the typical core cis-acting elements in promoter regions, including TATA and CAAT boxes. Potential regulatory cis-acting elements identified from the upstream region of the Ghrboh genes are shown in Fig. 6 and Additional file 1: Table S4. In addition to the TATA and CAAT boxes, there were 19 types of cis-acting elements, which could be grouped into four different functional categories, namely stress response, hormone regulation, cellular development, and metabolism regulation. These findings were consistent with those from a previous study in Arabidopsis and rice [12].

The results revealed that 7 types of stress-response elements, namely ARE, MBS, Box-W1, HSE, LTR, WUNmotif and TC-rich repeats, with responses to anaerobiosis, drought, fungal elicitors, heat stress, cold stress, wound stress, and defense stress, respectively, were identified in the Ghrboh promoter regions. Furthermore, 11 types of hormone regulation elements, namely ABRE, AuxRR-core, TGA-box, TGA-element, ERE, GAREmotif, TATC-box, P-box, CGTAC-motif, TGACG-motif and TCA-element, which were associated with abscisic acid (ABA), auxin (IAA), ethylene, gibberellin (GA), methyl jasmonate (MeJA) and salicylic acid (SA) responses, were found in the Ghrboh promoters. 


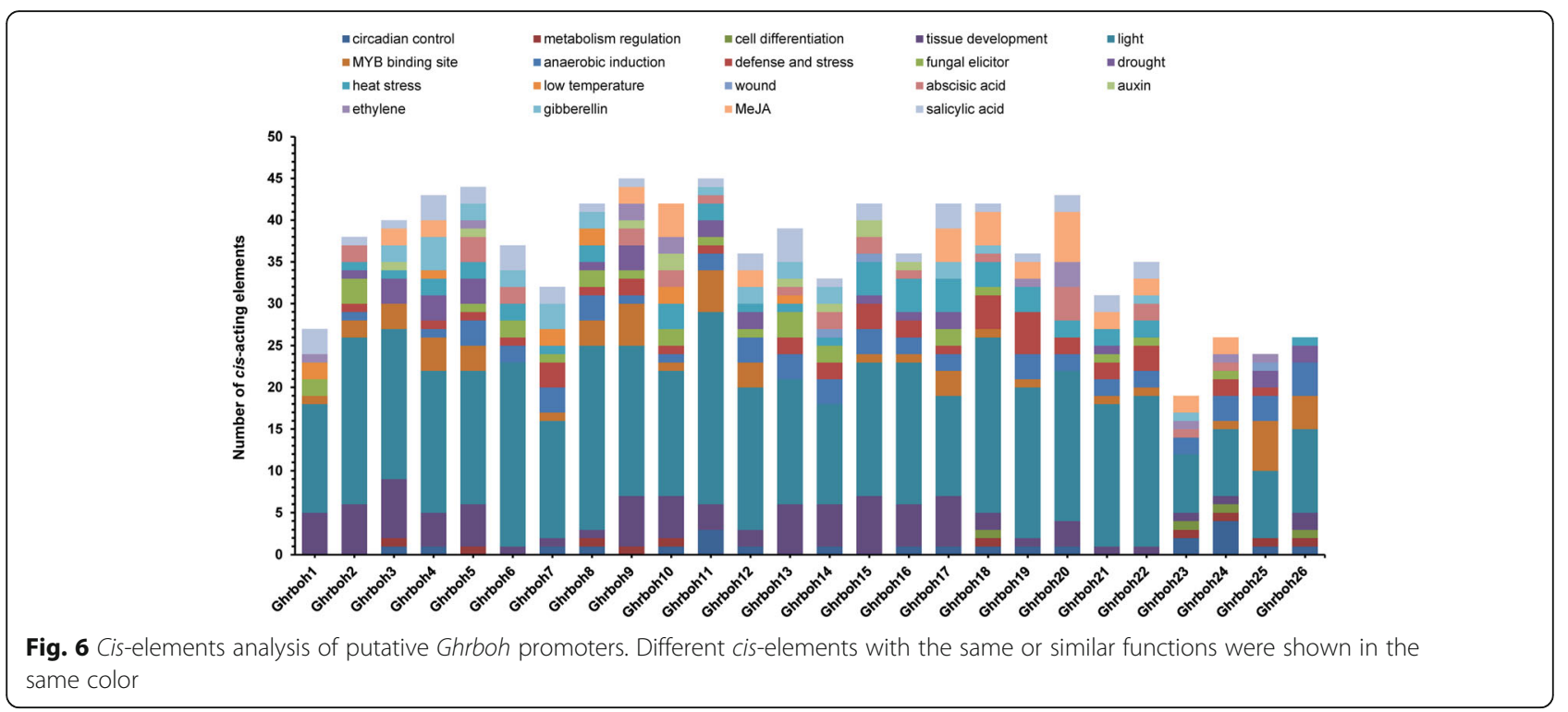

In the cellular development category, 7 types of cis-elements, namely HD-Zip 1, HD-Zip 2, Skn-1_motif, CATbox, RY-element, as-2-box and as1, which are associated with cell differentiation and tissue development, were identified in the Ghrboh promoter regions. In the metabolism regulation category, there were 4 types of elements, namely $\mathrm{O}_{2}$-site, MBSII, Unnamed_1 and circadian, which are associated with zein metabolism regulation, flavonoid biosynthesis gene regulation, phytochrome regulation and circadian control, respectively. In addition, many light-responsive elements were present in in each Ghrboh promoter. There were 29 different types of light-responsive elements and every putative promoter contained between six and 13 types (Fig. 6 and Additional file 1: Table S4). The putative promoters of Ghrboh genes carried different types and numbers of cis-regulatory elements, indicating that Ghrboh genes might be involved in some growth and development progresses, such as cotton fiber development, and were controlled by different regulatory mechanisms in response to various stresses.

\section{Predicting miRNA target sites}

To predict microRNA (miRNA)-mediated posttranscriptional regulation of Ghrbohs, we searched Ghrbohs coding sequences for target sites of G. hirsutum miRNAs, using the psRNATarget server with stricter parameters than default. The results showed that $15 \mathrm{G}$. hirsutum miRNAs targeted 17 Ghrbohs (Fig. 7, Additional file 1: Table S5). These miRNAs included conserved upland cotton miRNAs [32] and novel miRNAs identified by small-RNA sequencing and bioinformatics analysis [33,34]. The results showed that Ghrboh1 and Ghrboh2 were both targeted by ghr-miR3447 and novel_miR_2473 with sites in the
NADPH_Ox domain and the second EF-hand motif of the N-terminus, respectively; Ghrboh7 and Ghrboh8 were both targeted by ghr-miR1535a with sites in the NADPH_Ox domain; Ghrboh10 was targeted by ghr-miR3627c with a site in the NADPH_Ox domain; ghr-miR414b and ghrmiR838a targeted Ghrboh13 and/or Ghrboh14 with a site in the NAD-binding_6 domain; ghr-miR482d and ghrmiR838b both targeted Ghrboh15 and Ghrboh16 with the same sites in the FAD-binding_ 8 domain; ghr-miR2673 targeted Ghrboh 21 and Ghrboh22 with a site in the NADbinding_ 6 domain; ghr-miR482d and ghr-miR2595 targeted Ghrboh 23 and Ghrboh 24 with a site in the NADPH_Ox domain (Fig. 7). In addition to the target sites described above, other, novel miRNAs of upland cotton targeted Ghrbohs. Mar-F-3-m0087 targeted Ghrboh11 and Ghrboh12 with a site in the NADPH_Ox domain; and Mar-F-2-m0069 and ghr-miR2949a targeted Ghrboh17 and/or Ghrboh18 with a site in the Ferri_reduct domain or FAD-binding_8 domain, respectively (Additional file 1: Table S5). Our prediction results revealed that the miRNA-mediated posttranscriptional regulation of rbohs might be conserved in G. hirsutum, and many researchers have studied rboh genes involved in the process of morphogenesis and development, and response to biotic and abiotic stress in plants [10], but there have been few reports of gene expression and regulation being mediated by miRNAs. These miRNAs, predicted to target Ghrbohs, resulted from computational predictions and deep sequencing, and they were reported to be involved in some biological processes reported in plants, including responses to environmental stresses and regulation of cell growth and development [32, 34-38]. The expression patterns of the miRNAs mentioned above and their targets need to be detected and verified in further experiments to confirm and determine their biological functions 


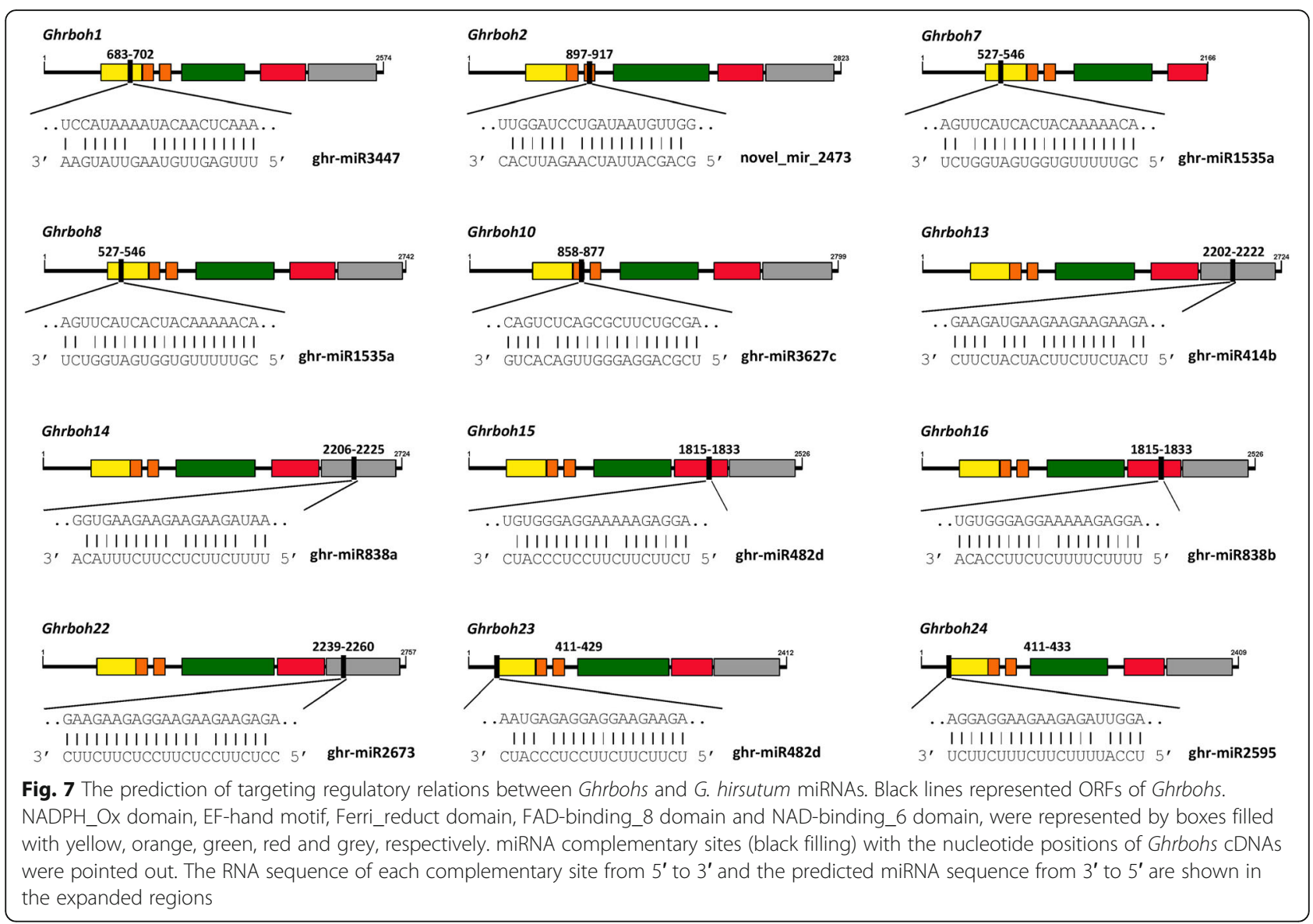

in upland cotton, and this is a topic on which we plan to report in greater detail in the future.

\section{Discussion}

The plant rboh gene family has been comprehensively analyzed in Arabidopsis, rice, grape, apple and rubber tree, respectively. However, there had been no genomescale analysis of the rboh gene family in upland cotton before the present study. In this study, the upland cotton rboh gene family was identified at the genome scale, and the expression patterns of individual members were analyzed.

\section{The $R$ boh gene family was expanding in upland cotton genome}

We identified 26 putative rboh genes (Ghrboh1 through Ghrboh26) from the genome of upland cotton cultivar TM-1. We also searched another 20 plant genomes for rboh genes, from lower aquatic to higher terrestrial plants, which were at key evolutionary nodes. The number of rboh genes in upland cotton is much larger than those from other plants, and the results showed that rboh gene family first appeared in green algae (C. reinhardtii, about four members) and the number dramatically increased in pteridophytes (S. moellendorffii, about ten members), then stayed relatively stable until the upland cotton evolved (G. hirsutum, having approximately twice the gene number of $S$. moellendorffii etc., and about six times the number of $C$. reinhardtii) (Additional file 1: Figure S1). The results showed that, as a result of plant evolution, the rboh gene family expanded.

Gene duplications, occurring during cotton evolution, have played a significant role in the expansion of the rboh gene family in the genome [26]. Genes may be duplicated by some mechanisms, such as WGD or polyploidy, tandem, proximal and/or dispersed duplication [27]. It is generally known that WGD or polyploidy are important processes throughout the history of plant evolution, and have long been recognized as fundamental mechanisms of diversification and gene family expansion in plants [39-42]. Throughout plant history, there have been some common WGD or polyploidy events, such as occurred at the appearance of the seed plants approximately 310 MYA and another paleohexaploidization event at the evolution of the eudicots 130 190 MYA, as well as some lineage-specific WGD or polyploidy events, such as the WGD series of the $\rho-\sigma-\tau$ in the cereal grass 
lineage and the $\alpha-\beta-\gamma$ series in the Arabidopsis lineage [43]. In the hypothetical origins and evolutionary history of dicotyledonous allotetraploid cotton, allopolyploid cotton may have appeared in the last 1 2 MYA, as a consequence of trans-oceanic dispersal of an A-genome taxon G. arboreum (A2) to the New World approximately 5 10 MYA, followed by hybridization with an indigenous D-genome diploid G. raimondii (D5), followed by chromosome doubling [29]. The results of the phylogenetic and evolution analysis showed that the paralogous pairs of Ghrbohs diverged approximately 8.34 MYA (Additional file 1: Table S2). The results of synteny and collinearity analysis revealed that the duplicate genes of the Ghrboh gene family were mainly duplicated by WGD or segmental duplications. These results suggested that the expansion of Ghrboh genes in upland cotton mostly arose from WGD or polyploidy events as one common ancestor diverged into G. raimondii and G. arboreum at approximately $2 \sim 13$ MYA. Thus, we hypothesized that the common and lineage-specific WGD or polyploidy events and segmental duplications, which generated duplicate copies of plant rboh genes and were widespread throughout plant history, are the major factor responsible for the expansion of the Ghrboh gene family.

\section{Ghrbohs probably participate in cotton fiber development and stress response by mediating ROS production}

There are various reports that suggest that NADPH oxidases mediate a multiplicity of physiological functions involved in development $[2,44,45]$, adaptation to environment [46-48], and interactions with other organisms $[49,50]$, and the expression patterns of rboh genes have been determined in many plant species. In apple, $M d r b o h D 1-3$ and $F$ were expressed in leaves, in vitro shoot and suspension cell cultures, and expression of MdrbohE2 and H1-2 varied among the tissues. The $M d r b o h D 1-2$ and $F$ genes were involved in regulation of developmental processes of apple shoots and in response to oxidative stress damage [16]. In rice, under drought stress, the expressions of OsNox1-3, OsNox5 and OsNox9 were up-regulated, but the expression of OsNox6 was down-regulated. Under high-temperature conditions, the expressions of OsNox5-9 were upregulated, but the expressions of OsNox1-3 were significantly down-regulated. Under salt stress, the expressions of OsNox 2 and $O s N o x 8$ were increased but the expressions of OsNox1/3/5/6 decreased [28]. In grape, the expression levels of $V v r b o h A / B / C 1$ were markedly induced by drought and salinity stresses. After powdery mildew inoculation, the expression of $\operatorname{vvb} b \mathrm{~B} B / C 2 / D$ increased while that of $V v r b o h H$ decreased [15]. These results suggest that the expression of plant rboh genes varied greatly with tissues and environmental conditions, suggesting diverse functions of rboh genes in the plant development and stress responses.

Although, cotton, which is a widely cultivated polyploid fiber crop, is a relatively salt and drought tolerant crop, exposure of cotton to high salinity or drought conditions can directly lead to a considerable negative impact on cotton growth and development and lint yield. To investigate the expression patterns of members of the Ghrboh gene family, we analyzed the transcript levels of all 26 Ghrboh genes in different organs/tissues, at different developmental stages, and following exposure to some abiotic stresses. From the results of transcriptomic data and qPCR, we found that the expression patterns of the rboh gene family of upland cotton exhibited diverse and complex stress-response expression signatures, which may be important both for stress responses and developmental processes.

To preliminarily explore which member(s) contributed mainly to the stress response or developmental processes, we analyzed the differential expression of the Ghrboh gene family in different tissues/organs, at different developmental stages of fiber/ovule, and under different abiotic stresses. Based on RNA-Seq data, the statistical significance of difference of gene expression was assessed with $\log 2$ (fold-change of Ghrbohs FPKMs (fragments per kilobase of transcript per million mapped reads) $) \geq 1$ and a $p$-value $<0.05$ (Fisher's Exact Test). It is worth noting that 3 genes (Ghrboh10/21/22) were significantly up-regulated during fiber and ovule development, 1 gene (Ghrboh26) was specifically responsive to salt stress and significantly expressed during fiber development, 1 gene (Ghrboh9) was significantly up-regulated during ovule development, and 6 genes (Ghrboh6/13/14/ $15 / 16 / 25)$ were significantly expressed in different tissues and organs (Additional file 1: Table S6 and Additional file 1: Figure S5).

The results of qPCR showed that some Ghrboh genes were specifically expressed at certain time points of fiber/ovule development and significantly induced by salt/drought stresses (Additional file 1: Figure S2 and Additional file 1: Figure S3). The results of digital expression were basically consistent with the results of the qPCR studies, although there were differences in the expression levels between our qPCR results and the RNASeq data. The differences might be because the material was collected from different tissues of different varieties at different growth stages. The material for qPCR was taken from G. hirsutum L. cv. SF06 at the appropriate stage, while the material for the RNA-seq was the leaf tissue of TM-1 (the age is not applicable). Despite this discrepancy, the findings suggested that these genes played an important role in the development of fibers and ovules. 
Interestingly, we also found that the expression patterns of Ghrboh genes in response to salinity was largely opposite to those obtained in response to drought stress. In other words, if the expression of a Ghrboh gene was upregulated under salinity stress, then expression of the gene was down-regulated under drought stress. Specifically, expression of Ghrboh2/10/11/14/20/22/23 was induced by salt but reduced by drought, whereas expression of Ghrboh4/7 was induced by salt but reduced by drought. Not all genes exposed to salinity and drought stresses showed this 'opposite trend': Ghrboh3 expression was upregulated by both salinity and drought stresses, whereas Ghrboh13 was significantly induced only by salinity (but not drought) at $12 \mathrm{~h}$, and Ghrboh12/21 was induced only by salinity stress (Additional file 1: Figure S4). The molecular mechanism of this interesting phenomenon needs to be studied further, a topic that we plan to report on in greater detail in the future.

These results suggested that the genes described above may be important for stress responses and/or developmental processes and will be useful in cloning candidate genes for functional analysis of their role in stress response and fiber development.

\section{The putative regulation mechanisms of Ghrboh gene expression}

As evident from a number of studies, NOX-dependent ROS production of plant is associated with numerous stress-, morphogenesis- and development-related signaling pathways, such as phytohormone signaling pathways [12], although how the ROS flux mediated by the Ghrboh gene family is deciphered downstream to achieve a specific response has yet to be elucidated. In the current study, the prediction of transcription-related components, including cis-elements and post-transcriptional regulation mediated by upland cotton miRNAs, may provide an insight into the putative regulatory mechanisms underlying Ghrboh gene expression and their functional multiplicity.

In the Ghrboh promoter regions, we found a number of stress-response elements, such as ARE, Box-W1, HSE, LTR, WUN-motif and TC-rich repeats, which are responsive to biotic and abiotic stresses (Fig. 6 and Additional file 1: Table S4). We also found several phytohormone regulatory elements in the Ghrboh promoters, which indicated that the Ghrboh gene family probably participates in phytohormonesignaling pathways. Specifically, we noted the ABRE, TGA/ AuxRR-core, ERE and GAREs elements, which were associated with $\mathrm{ABA}$, ethylene, gibberellin and auxin responses, respectively (Fig. 6 and Additional file 1: Table S4).

It is reported that ABA accumulates under stress conditions and plays an important role in the stress response and tolerance of plants, which may coordinate the ROS signaling route [51]. Several evidences show that ABA induces ROS accumulation in the apoplast, which is dependent on Rboh genes and plays an important role in ABA signaling [52]. For instance, in Arabidopsis, 2 Rboh genes (RbohD and F) of 10 functioning $R b o h$ genes $(R b o h A-H)$ had been shown to be involved in the ABA signaling [53]. In this study, the cis-acting element, ABA-responsive element (ABRE), was found in 15 Ghrboh genes promoter region (Fig. 6 and Additional file 1: Table S4). Based on the results of qPCR, we found that the vast majority of the 15 Ghrboh genes had significantly different expression patterns under drought and/ or salt stress (Additional file 1: Figure S4). In addition to the ABA, phytohormones, such as auxin [54, 55], ethylene $[56,57]$ and gibberellin [58] are known to play important roles in cotton fiber development. The development of cotton fiber includes four overlapping stages, which are defined based on the number of dpa: initiation ( -5 to $5 \mathrm{dpa}$ ), elongation ( 2 to $30 \mathrm{dpa}$; the most active elongation period is 5 to $20 \mathrm{dpa}$ ), secondary cell wall accumulation (20 to $50 \mathrm{dpa}$; the rapid accumulation period is 25 to $40 \mathrm{dpa}$ ) and maturation (45 to 60 dpa) [59-61]. Previous studies had revealed that auxin accumulates in the ovule epidermis and fiber cells from - 5 to $10 \mathrm{dpa}$ [55], and a substantial amount of ethylene and gibberellin were synthesized in the elongating fiber cells, with the biosynthesis of ethylene and gibberellin being two of the most significantly upregulated biochemical pathways during cotton fiber elongation [56, 62]. In the current study, based on the results of qPCR, we analyzed the expression patterns of those Ghrboh genes that carried the phytohormone-responsive elements in the promoters in both fibers and ovules during cotton fiber development, and found that the vast majority of them had significantly different expression patterns from one another at the corresponding time points of phytohormone accumulation during cotton fiber development. For instance, among the genes associated with auxin response, Ghrboh3 showed significantly up-regulated expression at $10 \mathrm{dpa}$ in the fiber and at -1 and $0 \mathrm{dpa}$ in the ovule, whereas Ghrboh13 and Ghrboh14 showed significantly up-regulated expression at $5 \mathrm{dpa}$ in the fiber and from 5 to $10 \mathrm{dpa}$ in the ovule (Additional file 1: Figure S2 and Additional file 1: Figure S3). Among the genes associated with ethylene response, Ghrboh5 showed significantly down-regulated expression in the fiber during the most active fiber elongation period, from 5 to $20 \mathrm{dpa}$, whereas Ghrboh9 showed significantly up-regulated expression from 5 to 10 dpa in the fiber, Ghrboh 23 showed significantly up-regulated expression at 5 and $20 \mathrm{dpa}$ in the fiber andGhrboh10/24/25 all exhibited significantly upregulated expression at $5 \mathrm{dpa}$ in the fiber (Additional file 1: Figure S3). Among the genes associated with gibberellin response, Ghrboh6 showed significantly down-regulated expression from 5 to $20 \mathrm{dpa}$ in fiber, Ghrboh7/22/23 showed significantly up-regulated expression at 5 and 20 
dpa in the fiber and Ghrboh8/12 showed significantly upregulated expression from 10 to $20 \mathrm{dpa}$ in the fiber (Additional file 1: Figure S3). These results suggested that these genes are probably responsive to phytohormones, and the Ghrboh gene family might be regulated by the cis-elements associated with phytohormone signaling during cotton fiber development and stress responses.

We also predicted miRNA-mediated posttranscriptional regulation of Ghrbohs and identified some putative target sites of upland cotton miRNAs. These miRNAs were divided into a conserved group (e.g. ghr-miR414, ghr-miR482, ghr-miR2949 and ghrmiR3627) and a novel group (e.g. novel_mir_2473, MarF-3-m0087 and ghr-miR2673). Previous studies had indicated that these conserved and novel miRNAs were involved in some biological processes, including responses to environmental stresses and regulation of cell growth, development and metabolism in association with cotton fiber development [32-35, 63, 64]. For instance, ghrmiR2949, ghr-miR3627 and novel_mir_2473 have been proposed to be involved in cotton fiber development [33], whereas ghr-miR414 and Mar-F-3-m0087 might be associated with stress response and genetic male-sterility in upland cotton $[34,65]$, respectively. Our results will help point us in the appropriate direction for further experiments to determine the biological functions of these miRNAs and their targets in upland cotton.

Plants respond to environmental stress and regulate growth and development in multiple ways and have evolved mechanisms to increase their tolerance to abiotic stresses and to modulate relevant metabolism processes through interactive molecular and cellular changes. These mechanisms involve multiple systems, the foundation of which is a cooperative action of signal cascade transduction networks, involving multiple genes. However, evidence on the upstream regulation of Ghrbohs and the downstream factors regulated by Ghrbohs at different levels is lacking. For instance, one of the mechanisms that contributes to ROSinduced pathogen tolerance is the activation of many enzymatic and nonenzymatic antioxidants, such as glutathione-S-transferases (GSTs), ascorbate peroxidases (APXs), superoxide dismutases (SODs), catalases (CATs), glutathione and ascorbic acid [66]. But the relationships between the innate plant immune system and the activation of antioxidants, as well as non-coding RNA-mediated stress tolerance in plant, needs further investigation.

\section{Conclusions}

We identified 26 putative rboh genes distributed over 18 of the 26 chromosomes or scaffolds in the upland cotton genome. During the evolutionary process, WGD or polyploidy events and segmental duplications contributed to the expansion of the Ghrboh gene family. The expression patterns of the Ghrboh gene family were analyzed using
RNA-Seq and qPCR and showed different expression patterns in different tissues/organs, at different developmental stages and under different stresses, indicating diverse functions in growth, development and stress response of cotton. The promoter sequence analysis revealed that there were many cis-acting elements associated with phytohormone and stress response, but different members harbored distinct types and numbers, which suggested that individual members of the Ghrboh gene family might be differentially regulated at the transcriptional level. Moreover, we also predicted and analyzed the miRNA-mediated post-transcriptional regulation of the gene family in this species. Taking all these results into account, we hypothesized that the Ghrboh gene family, which might be regulated by cis-elements and miRNAs at different levels, played roles in cotton development and stress tolerance through modulating NOX-dependent ROS induction. Collectively, our study provides a comprehensive analysis of and novel insights into the expression, regulation, and evolution of the Ghrboh gene family, and helps lay the foundation for further cloning and functional verification of the Ghrboh genes by reverse genetics research. Additionally, these results may increase our understanding of the molecular basis of many important traits in agronomic upland cotton, such as fiber development, pathogen resistance, and tolerance to abiotic stresses.

\section{Methods}

\section{Identification of Rboh genes}

The upland cotton genome files (G. hirsutum, NAU) were downloaded from the Cotton Functional Genomics Database (CottonFGD) (https://cottonfgd.org/) [67]. To identify the rboh genes in upland cotton, the BLAST algorithm for Proteins (BLASTP) [68] was performed using the fulllength protein sequences coded by ten $r b o h$ genes from $A$. thaliana (Locus ID see Additional file 1: Table S3) and the Hidden Markov Model (HMM) profile of the NADPH_Ox (PF08414), EF-hand (PF00036), Ferric reductase NAD binding domain (PF08030) and FAD-binding domain (PF08022) obtained from Pfam (http://pfam.xfam. org/) [69] as the queries. InterProScan (version 4.8) [70] was further used to confirm the inclusion of the conserved domain of rboh in each candidate sequence using the Pfam database (http://pfam.xfam.org/). The rboh genes of the other 20 plant genomes obtained from the JGI database (http://www.phytozome.net) [71] and CottonGen (https://www.cottongen.org) [72] (Additional file 1: Figure S1) were identified using methods similar to those described above. The details of upland cotton rboh (Ghrboh) genes, including locus ID, genomic position, gene length and open reading frames length, were collected from the G. hirsutum genome and annotation files. 


\section{Sequence and functional annotation analysis}

The graphical visualization of Ghrboh genes exons-intron structures gathered from the GFF3 file of the upland cotton genome was performed by the Gene Structure Display Server (GSDS) (http://gsds.cbi.pku.edu.cn/) [73]. The ProtParam tool was used to calculate the physicochemical characteristics of Ghrboh proteins (http://www. expasy.org/tools/protparam.html), including the number of amino acids, molecular weight, instability index and theoretical isoelectric point. Predictions of subcellular localizations of Ghrboh proteins were performed with CELLO v.2.5 (http://cello.life.nctu.edu.tw/) [74]. The conserved domains of all the protein sequences coded by candidate Ghrbohs were predicted with the Simple Modular Architecture Research Tool (SMART) (http://smart.emblheidelberg.de/) [75]. The IBS software (http://ibs.biocuckoo.org/) [76], called illustrator for biological sequences, was used for preparing the Ghrboh protein functional domain graphs. Prediction of transmembrane helices in predicted upland cotton rboh proteins were performed with the TMHMM Server v. 2.0 (http://www.cbs. dtu.dk/services/TMHMM/).

\section{Synteny and collinearity analysis}

The chromosomal location of rboh genes was drafted from top to bottom on upland cotton chromosomes according to gene positions in the genome annotation by Circos-0.69 (http://circos.ca/) [77]. A synteny analysis was conducted locally using a method similar to that developed for the Plant Genome Duplication Database (http:// chibba.pgml.uga.edu/duplication/) [78]. We used BLAST+ version 2.6.0 [68] for the pairwise comparison of the filtered rboh protein sets of G. hirsutum, G. raimondii and G. arboreum. Then, MCscanX [79] was employed to identify homologous regions, and syntenic blocks and duplicate gene classifications were evaluated using Circos-0.69. Default parameters were used in all the steps.

\section{Phylogenetic and evolutionary analysis}

The sequence data used in this study were collected from the National Center for Biotechnology Information (NCBI) (http://www.ncbi.nlm.nih.gov/protein/) and the JGI database (http://www.phytozome.net) [71]. The fulllength coding sequences of the plant rboh genes were aligned by the ClustalW program with default parameters [80]. MEGA6.0 software was used to construct the phylogenetic trees with a bootstrap analysis of 1000 replicates and the neighbor-joining (NJ) method [81]. In addition, to further estimate Ghrboh genes duplication events, the non-synonymous $(K a)$ and synonymous $(K s)$ substitution rates of evolution were calculated using add_ka_and_ks_to_collinearity, the downstream analysis program of the MCScanX package [79]. To estimate the evolutionary duplication time of duplicated genes, Ks values were translated into duplication time in millions of years based on a rate of one substitution per synonymous site per year. The duplication events time $(t)$ was calculated from the equation " $t=K s / 2 r$ ", where " $r$ " was the neutral substitution rate. A neutral substitution rate of $2.6 \times 10^{-9}$ was used in the current study [31].

\section{Digital expression profiling analysis}

Expression value (FPKMs) of Ghrbohs was obtained from the websites at CottonFGD (https://cottonfgd.org/) [67] and ccNET (http://structuralbiology.cau.edu.cn/gossypium/) [82]. We determined the expression differences of Ghrbohs in different tissues/organs (root, stem, leaf, petal, torus, stamen, pistil and calycle), at different developmental processes of ovules $(-3,-1,0,1,3,5,10,20$, 25 and $35 \mathrm{dpa})$ and fibers (5, 10, 20 and $25 \mathrm{dpa})$, and under different stress treatments (hot, cold, PEG and salt treatments) with $\log _{2}$ (fold-change of Ghrbohs FPKMs) $\geq 1$ and $p$-value $<0.05$ (Fisher's Exact Test).

\section{Plant materials and stress treatments}

The G. hirsutum cv. SF06 plants were used in this research and were cultivated in a trial field from April to September under standard conditions in Tai'an, the experimental station of Shandong Agricultural University. Flowers were tagged on the day of anthesis, and cotton bolls were harvested at $0,3,5,10,20$ and 25 days postanthesis (dpa); the bolls at -1 dpa were harvested based on the characteristics of cotton budding. We excised ovules from the bolls, and scraped fibers from the ovules at 5, 10, 20 and $25 \mathrm{dpa}$. All ovules and fibers were frozen in liquid nitrogen and stored at $-80{ }^{\circ} \mathrm{C}$ until total RNA was extracted. For the stress treatments, the seeds of upland cotton were sown in a soil mix [peat moss:perlite, $2: 1(\mathrm{v} / \mathrm{v})]$ in plastic pots and were placed in plant growth chambers under the following conditions: $28^{\circ} \mathrm{C} / 21^{\circ} \mathrm{C}$ day/night temperature, $16 / 8 \mathrm{~h}$ light/dark photoperiod, $3300 \mathrm{~lx}$ light intensity and a relative humidity of $70 \%$. And, the uniform-sized plantlets were cultivated in Hoagland's solution $(\mathrm{pH}=5.6$ and changing every $3 \mathrm{~d}$ ) after the expansion of the first true leaf. Approximately one week later, the plantlets were treated, with the nutrient solution supplemented with $250 \mathrm{mM} \mathrm{NaCl}$ for the salt treatment, or $20 \%(\mathrm{v} / \mathrm{v})$ polyethylene glycol (PEG) 6000 for the drought treatment. The leaves of treated plantlets were harvested after $0,1,3,6$ and $12 \mathrm{~h}$ stress treatment. All the harvested samples were frozen in liquid nitrogen and stored at $-80^{\circ} \mathrm{C}$ until total RNA was extracted. Three independent biological replicates were performed for each treatment.

\section{Quantitative polymerase chain reaction (qPCR)}

Total RNA from these samples was isolated using RNAprep Pure Plant Kit (Polysaccharides \& Polyphenolics- 
rich, DP441) (TIANGEN, Beijing, China). The quality and concentrations of the isolated RNA samples were determined by $1.5 \%$ agarose gel electrophoresis and a NanoDrop 2000 Spectrophotometer (Thermo Fisher Scientific, Wilmington, DE, USA), respectively. Reverse transcription PCR was carried out using HiScript $^{\circ}$ II Q RT SuperMix for qPCR with gDNA wiper (R223) (Vazyme, Nanjing, China). Transcript levels were determined using a QuantStudio ${ }^{\mathrm{Tw}} 6$ Flex Real-Time PCR System (Applied Biosystems ${ }^{\mathrm{Tw}}$, Carlsbad, CA, USA) and ChamQTM Universal SYBR $^{\circ}$ qPCR Master Mix (Q711) (Vazyme), with three technical replicates for each biological sample. PCR thermal cycling included an initial denaturation at $95^{\circ} \mathrm{C}$ for $1 \mathrm{~min}$, followed by 40 cycles at $95^{\circ} \mathrm{C}$ for $10 \mathrm{~s}$ and $60^{\circ} \mathrm{C}$ for $30 \mathrm{~s}$ in a reaction volume of $20 \mu \mathrm{l}$ in $0.1 \mathrm{ml}$ MicroAmp $^{\text {Th }}$ Fast Optical 96-Well Reaction Plate (4346907) (Applied Biosystems). Following the PCR, a melting curve analysis was performed. Cycle threshold was used for the relative quantification of the input target number. Relative fold difference represents the number of treated target gene transcript copies relative to the number of untreated gene transcript copies, and was calculated according to the $2^{-\Delta \Delta C T}$ method [83]. To normalize the variance among samples, cotton ubiquitin 7 (UBQ7) was used as an endogenous control. Gene-specific primers used for qPCR were designed using Primer Premier 5.0 [84] and are listed in Additional file 1: Table S7. For statistical analysis, standardization of gene expression data was repeated at least three times with three biological replicates [85]. ANOVA (analysis of variance) was calculated using DPS (version 7.05) [86], and, if significant, the differences between samples were compared by LSD's test $(p<0.05)$.

\section{Prediction of Ghrbohs regulatory elements}

The genomic sequences at $1.5-\mathrm{kb}$ upstream of the translation start site (TSS) of each Ghrboh gene were extracted from the genome files of G. hirsutum TM-1. The PlantCARE serve was used to predict the transcriptional response elements of Ghrboh gene promoters (http://bioinformatics.psb.ugent.be/webtools/plantcare/html/) [87]. We obtained cotton miRNA sequences from miRBase (http://www.mirbase.org/) [88], the Plant MicroRNA database (http://bioinformatics.cau.edu.cn/PMRD/) [89], the Cotton EST database (http://www.ncbi.nlm.nih.gov/nucest) and published articles. Ghrboh genes targeted by miRNAs were predicted by searching coding sequences (CDS) regions for sequences complementary to the cotton miRNAs, using the psRNATarget server with default parameters, except for maximum expectation $(E)=3.0$ and maximum unpaired energy $(\mathrm{UPE})=20.0$ (http://plantgrn.noble.org/ psRNATarget/home) [90].

\section{Supplementary information}

Supplementary information accompanies this paper at https://doi.org/10. 1186/s12864-020-6503-6.

Additional file 1: Table S1. Orthologous rboh gene pairs of $G$. hirsutum, G. arboreum, and G. raimondii. Table S2. The non-synonymous (Ka) and synonymous (Ks) substitution and estimated age of the duplication events for Ghrboh paralogous genes. Table S3. Gene numbers of rboh gene family in 17 plant genomes. Table S4. List of identified cis-elements in the putative promoter region of 26 Ghrboh genes using PlantCARE web tool. Table S5. The details of predicted targeting regulatory relations between Ghrbohs and G. hirsutum miRNAs using psRNATarget web server. Table S6. The details of Ghrbohs expression difference in different tissues and/ororgans, at developmental processesof ovules and fibers, and under different stress treatments. Table S7. Gene-specific primers used for qPCR analysis of Ghrboh genes. Figure S1. Comparisons of rboh gene numbers across a wide range of organisms. Figure S2. Relative transcriptional expression levels of Ghrbohs in different developmental stages of upland cotton fiber by qPCR Figure S3. Relative transcriptional expression levels of Ghrbohs in different developmental stages of upland cotton ovule by qPCR. Figure S4. Relative transcriptional expression levels of Ghrboh under NaCl and PEG treatments by qPCR. Figure S5. Venn diagram analysis of Ghrbohs expression difference.

\section{Abbreviations}

aa: Amino acid; Ac: Ananas comosus; Amt: Amborella trichopoda; ARE: Anaerobic responsive element; At: Arabidopsis thaliana; Bd: Brachypodium distachyon; bp: Base pair; CDS: Coding sequence; Cr: Chlamydomonas reinhardtii; Dc: Daucus carota; DOA: Day of anthesis; DPA: Days post-anthesis; ERE: Ethylene-responsive element; FPKMs: Fragments per kilobase of transcript per million mapped reads; Ga: Gossypium arboretum; GAREs: GA-responsive elements; Gh: Gossypium hirsutum; Gr: Gossypium raimondii; HMM: Hidden Markov Model; HSE: Heat stress element; kb: Kilobase; LTR: Low-temperature responsiveness; Ma: Musa acuminata; Md: Malus domestica; miRNA: MicroRNA; Mp: Micromonas pusilla; Mt: Medicago truncatula; MW: Theoretical molecular weight of proteins; MYA: Million years ago; NADPH: Nicotinamide adenine dinucleotide phosphate; NOX: NADPH oxidase; nt: Nucleotide; Ol: Ostreococcus lucimarinus; ORF: Open reading frame; Os: Oryza sativa; PCD: Programmed cell death; PCR: Polymerase chain reaction; PCW: Primary cell wall; pl: Isoelectric point; Pp: Physcomitrella patens; qRT-PCR: Quantitative reverse-transcription polymerase chain reaction; rbohs: Respiratory burst oxidase homologs; ROS: Reactive oxygen species; Sb: Sorghum bicolor; SCW: Secondary cell wall; Si: Setaria italica; SOD: Superoxide dismutase; Ta: Triticum aestivum; TC: Theobroma cacao; TF: Transcription factors; TFBS: Transcription factor binding sites; UBQ: Ubiquitin extension protein; UTR: Untranslated regions; Vc: Volvox carteri; VV: Vitis vinifera; WGD: Whole genome duplication; WGT: Whole genome triplication; Zm: Zea mays

\section{Acknowledgements}

We thank Dr. Zujun Yin of the Institute of Cotton Research, Chinese Academy of Agricultural Sciences, for providing valuable advice on bioinformatics analysis.

\section{Authors' contributions}

WW and FS conceived and designed the research; WW, DC, DL, YC, JD, MH, $\mathrm{LS}$ and $\mathrm{XZ}$ performed the experiments; WW analyzed the data and prepared figures; DC and DL contributed analysis tools; WW and YC wrote the manuscript; FS performed English editing. All authors have read and approved the final manuscript.

\section{Funding}

This work was supported by the National Key R\&D Program of China (2018YFD0100303) and the China Major Projects for Transgenic Breeding (2016ZX08005-004). The funding agency played no role in the design of the study; in the collection, analyses, or interpretation of data; or in the writing of the manuscript, or in the decision to publish the result. 


\section{Availability of data and materials}

All analysis results data generated during this study were included in this article and its Additional data repository.

\section{Ethics approval and consent to participate}

All the cotton materials analyzed for this study were collected from the State Key Laboratory of Crop Biology, Shandong Agricultural University, which were public and available for non-commercial purpose. This article did not contain any studies with human participants or animals performed by any of the authors.

\section{Consent for publication}

Not applicable.

\section{Competing interests}

The authors declare no conflict of interest.

\section{Received: 19 July 2019 Accepted: 16 January 2020}

Published online: 29 January 2020

\section{References}

1. Swanson S, Gilroy S. ROS in plant development. Physiol Plant. 2010;138(4): 384-92.

2. Xie H-T, Wan Z-Y, Li S, Zhang Y. Spatiotemporal production of reactive oxygen species by NADPH oxidase is critical for Tapetal programmed cell death and pollen development in Arabidopsis. Plant Cell. 2014;26(5):200723.

3. Kwak JM, Nguyen $V$, Schroeder JI. The role of reactive oxygen species in hormonal responses. Plant Physiol. 2006;141(2):323-9.

4. Torres MA, Dangl $\mathrm{J}$. Functions of the respiratory burst oxidase in biotic interactions, abiotic stress and development. Curr Opin Plant Biol. 2005;8(4): 397-403.

5. Xu J, Wang G, Wang J, Li Y, Tian L, Wang X, Guo W. The lysin motifcontaining proteins, Lyp1, Lyk7 and LysMe3, play important roles in chitin perception and defense against Verticillium dahliae in cotton. BMC Plant Biol. 2017;17(1):148.

6. Mittler R. ROS are good. Trends Plant Sci. 2016;22(1):11-9.

7. Wang W, Chen D, Zhang X, Liu D, Cheng Y, Shen F. Role of plant respiratory burst oxidase homologs in stress responses. Free Radic Res. 2018;52(8):82639.

8. Oda T, Hashimoto H, Kuwabara N, Akashi S, Hayashi K, Kojima C, Wong HL, Kawasaki T, Shimamoto K, Sato M, et al. Structure of the N-terminal regulatory domain of a plant $\mathrm{NADPH}$ oxidase and its functional implications. J Biol Chem. 2010;285(2):1435-45.

9. Groom QJ, Torres MA, Fordham-Skelton AP, Hammond-Kosack KE, Robinson $\mathrm{NJ}$, Jones JDG. rbohA, a rice homologue of the mammalian gp91phox respiratory burst oxidase gene. PIJ. 1996;10(3):515-22.

10. Kaur G, Sharma A, Guruprasad K, Pati PK. Versatile roles of plant NADPH oxidases and emerging concepts. Biotechnol Adv. 2014;32(3):551-63.

11. Sagi M, Fluhr R. Production of reactive oxygen species by plant NADPH oxidases. Plant Physiol. 2006;141(2):336-40.

12. Kaur G, Pati PK. Analysis of cis-acting regulatory elements of respiratory burst oxidase homolog (Rboh) gene families in Arabidopsis and rice provides clues for their diverse functions. Comput Biol Chem. 2016;62:104-18.

13. J. Lightfoot D, Boettcher A, Little A, Shirley N, Able AJ. Identification and characterisation of barley (Hordeum vulgare) respiratory burst oxidase homologue family members. Funct Plant Biol 2008; 35(5):347-359.

14. Marino D, Andrio E, Danchin EGJ, Oger E, Gucciardo S, Lambert A, Puppo A, Pauly N. A Medicago truncatula NADPH oxidase is involved in symbiotic nodule functioning. New Phytol. 2011;189(2):580-92.

15. Cheng C, Xu X, Gao M, Li J, Guo C, Song J, Wang X. Genome-wide analysis of respiratory burst oxidase homologs in grape (Vitis vinifera L.). Int J Mol Sci. 2013;14(12):24169-86.

16. Cepauskas D, Miliute I, Staniene G, Gelvonauskiene D, Stanys V, Jesaitis AJ, Baniulis D. Characterization of apple NADPH oxidase genes and their expression associated with oxidative stress in shoot culture in vitro. PCTOC. 2016;124(3):621-33.

17. Zou Z, Yang J, Zhang X. Insights into genes encoding respiratory burst oxidase homologs (RBOHs) in rubber tree (Hevea brasiliensis Muell. Arg.). Ind Crop Prod. 2019;128:126-39.
18. Yang C-Y, Hong C-P. The NADPH oxidase Rboh D is involved in primary hypoxia signalling and modulates expression of hypoxia-inducible genes under hypoxic stress. Environ Exp Bot. 2015;115:63-72.

19. Lin I-S, Wu Y-S, Chen C-T, Chen G-H, Hwang S-G, Jauh G-Y, Tzen JTC, Yang $\mathrm{C}-\mathrm{Y}$. AtRBOH I confers submergence tolerance and is involved in auxinmediated signaling pathways under hypoxic stress. Plant Growth Regul. 2017;83(2):277-85

20. Chen ZJ, Scheffler BE, Dennis E, Triplett BA, Zhang T, Guo W, Chen X, Stelly DM, Rabinowicz PD, Town CD, et al. Toward sequencing cotton (Gossypium) genomes. Plant Physiol. 2007;145(4):1303-10.

21. Tang W, Tu L, Yang X, Tan J, Deng F, Hao J, Guo K, Lindsey K, Zhang X. The calcium sensor GhCaM7 promotes cotton fiber elongation by modulating reactive oxygen species (ROS) production. New Phytol. 2014;202(2):509-20.

22. Li F, Fan G, Lu C, Xiao G, Zou C, Kohel RJ, Ma Z, Shang H, Ma X, Wu J, et al. Genome sequence of cultivated upland cotton (Gossypium hirsutum TM-1) provides insights into genome evolution. Nat Biotech. 2015;33(5):524-30.

23. Wang K, Wang Z, Li F, Ye W, Wang J, Song G, Yue Z, Cong L, Shang H, Zhu $\mathrm{S}$, et al. The draft genome of a diploid cotton Gossypium raimondii. Nat Genet. 2012;44(10):1098-103.

24. Zhang T, Hu Y, Jiang W, Fang L, Guan X, Chen J, Zhang J, Saski CA, Scheffler BE, Stelly DM, et al. Sequencing of allotetraploid cotton (Gossypium hirsutum L. acc. TM-1) provides a resource for fiber improvement. Nat Biotechnol. 2015;33(5):531-7.

25. Li F, Fan G, Wang K, Sun F, Yuan Y, Song G, Li Q, Ma Z, Lu C, Zou C, et al. Genome sequence of the cultivated cotton Gossypium arboreum. Nat Genet. 2014;46(6):567-72

26. Rong J, Feltus FA, Liu L, Lin L, Paterson AH. Gene copy number evolution during tetraploid cotton radiation. Heredity (Edinb). 2010;105(5):463-72.

27. Freeling M. Bias in plant gene content following different sorts of duplication: tandem, whole-genome, segmental, or by transposition. Annu Rev Plant Biol. 2009;60:433-53.

28. Wang G-F, Li W-Q, Li W-Y, Wu G-L, Zhou C-Y, Chen K-M. Characterization of Rice NADPH oxidase genes and their expression under various environmental conditions. Int J Mol Sci. 2013;14(5):9440.

29. Wendel J, Brubaker C, Alvarez I, Cronn R, Stewart J. Evolution and Natural History of the Cotton Genus. In: Genetics and Genomics of Cotton. Edited by Paterson A, vol. 3: Springer US; 2009: 3-22.

30. Hurst LD. The Ka/Ks ratio: diagnosing the form of sequence evolution. Trends Genet. 2002;18(9):486.

31. Senchina DS, Alvarez I, Cronn RC, Liu B, Rong J, Noyes RD, Paterson AH, Wing RA, Wilkins TA, Wendel JF. Rate variation among nuclear genes and the age of polyploidy in Gossypium. Mol Biol Evol. 2003;20(4):633-43.

32. Xie F, Zhang B. microRNA evolution and expression analysis in polyploidized cotton genome. Plant Biotechnol J. 2015;13(3):421-34.

33. Xue W, Wang Z, Du M, Liu Y, Liu JY. Genome-wide analysis of small RNAs reveals eight fiber elongation-related and 257 novel microRNAs in elongating cotton fiber cells. BMC Genomics. 2013;14(1):629.

34. Wei M, Wei H, Wu M, Song M, Zhang J, Yu J, Fan S, Yu S. Comparative expression profiling of miRNA during anther development in genetic male sterile and wild type cotton. BMC Plant Biol. 2013;13:66.

35. Guleria P, Yadav SK. Identification of miR414 and expression analysis of conserved miRNAs from Stevia rebaudiana. Genomics Proteomics Bioinformatics. 2011;9(6):211-7.

36. Macovei A, Tuteja N. microRNAs targeting DEAD-box helicases are involved in salinity stress response in rice (Oryza sativa L.). BMC Plant Biol. 2012;12: 183.

37. He Q, Zhu S, Zhang B. MicroRNA-target gene responses to lead-induced stress in cotton (Gossypium hirsutum L.). Funct Integr Genomics. 2014;14(3): 507-15.

38. Zhang Y, Wang W, Chen J, Liu J, Xia M, Shen F. Identification of miRNAs and their targets in cotton inoculated with Verticillium dahliae by highthroughput sequencing and Degradome analysis. Int J Mol Sci. 2015;16(7): 14749.

39. Segraves KA. The effects of genome duplications in a community context. New Phytol. 2017;215(1):57-69.

40. Wang W, Zhang X, Deng F, Yuan R, Shen F. Genome-wide characterization and expression analyses of superoxide dismutase (SOD) genes in Gossypium hirsutum. BMC Genomics. 2017:18(1):376.

41. Chai C, Wang Y, Valliyodan B, Nguyen HT. Comprehensive analysis of the soybean (Glycine max) GmLAX auxin transporter gene family. Front Plant Sci. 2016;7. 
42. Chen J, Zhang Y, Liu J, Xia M, Wang W, Shen F. Genome-wide analysis of the RNA helicase gene family in Gossypium raimondii. Int J Mol Sci. 2014; 15(3):4635-56.

43. Jiao $\mathrm{Y}, \mathrm{Li}$ J, Tang H, Paterson AH. Integrated syntenic and phylogenomic analyses reveal an ancient genome duplication in monocots. Plant Cell. 2014;26(7):2792-802.

44. Mangano S, Denita-Juarez SP, Choi H-S, Marzol E, Hwang Y, Ranocha P, Velasquez SM, Borassi C, Barberini ML, Aptekmann AA, et al. Molecular link between auxin and ROS-mediated polar growth. Proc Natl Acad Sci. 2017; 114(20):5289-94.

45. Kaya H, Iwano M, Takeda S, Kanaoka MM, Kimura S, Abe M, Kuchitsu K. Apoplastic ROS production upon pollination by RbohH and RbohJ in Arabidopsis. Plant Signal Behav. 2015;10(2):e989050.

46. Kolupaev YE, Firsova EN, Yastreb TO. Induction of plant cells heat resistance by hydrogen sulfide donor is mediated by $\mathrm{H}_{2} \mathrm{O}_{2}$ generation with participation of NADPH oxidase and superoxide dismutase. Ukrainian Biochem J. 2017:(4):34-42

47. Xie Y-J, Xu S, Han B, Wu M-Z, Yuan X-X, Han Y, Gu Q, Xu D-K, Yang Q, Shen W-B. Evidence of Arabidopsis salt acclimation induced by up-regulation of HY1 and the regulatory role of RbohD-derived reactive oxygen species synthesis. PL. 2011;66(2):280-92.

48. Rawat N, Chiruvuri Naga N, Raman Meenakshi S, Nair S, Bentur JS. A novel mechanism of gall midge resistance in the rice variety Kavya revealed by microarray analysis. Funct Integr Genomics. 2012;12(2):249-64.

49. Zhao YL, Zhou T, Guo HS. Hyphopodium-specific VdNoxB/NdPIs1dependent ROS- $\mathrm{Ca}^{2+}$ signaling is required for plant infection by Verticillium dahliae. PLoS Pathog. 2016:12(7):e1005793.

50. Chaouch S, Queval G, Noctor G. AtRbohF is a crucial modulator of defenceassociated metabolism and a key actor in the interplay between intracellular oxidative stress and pathogenesis responses in Arabidopsis. PLJ. 2012;69(4):613-27.

51. Yoshida T, Mogami J, Yamaguchi-Shinozaki K. ABA-dependent and ABAindependent signaling in response to osmotic stress in plants. Curr Opin Plant Biol. 2014;21:133-9.

52. Zhou J, Wang J, Li X, Xia X-J, Zhou Y-H, Shi K, Chen Z, Yu J-Q. $\mathrm{H}_{2} \mathrm{O}_{2}$ mediates the crosstalk of brassinosteroid and abscisic acid in tomato responses to heat and oxidative stresses. J Exp Bot. 2014;65(15):4371-83.

53. Toum L, Torres PS, Gallego SM, Benavídes MP, Vojnov AA, Gudesblat GE. Coronatine Inhibits Stomatal Closure through Guard Cell-Specific Inhibition of NADPH Oxidase-Dependent ROS Production. Front Plant Sci. 2016; 7(1851).

54. Zhang M, Zheng X, Song S, Zeng Q, Hou L, Li D, Zhao J, Wei Y, Li X, Luo M, et al. Spatiotemporal manipulation of auxin biosynthesis in cotton ovule epidermal cells enhances fiber yield and quality. Nat Biotechnol. 2011;29: 453.

55. Chen ZJ, Guan X. Auxin boost for cotton. Nat Biotechnol. 2011;29(5):407-9.

56. Shi Y-H, Zhu S-W, Mao X-Z, Feng J-X, Qin Y-M, Zhang L, Cheng J, Wei L-P, Wang Z-Y, Zhu Y-X. Transcriptome profiling, molecular biological, and physiological studies reveal a major role for ethylene in cotton Fiber cel elongation. Plant Cell. 2006;18(3):651-64.

57. Naoumkina M, Bechere E, Fang DD, Thyssen GN, Florane CB. Genome-wide analysis of gene expression of EMS-induced short fiber mutant Ligon lintless-y (liy) in cotton (Gossypium hirsutum L.). Genomics. 2017;109(3-4): 320-9.

58. Xiao G-H, Wang K, Huang G, Zhu Y-X. Genome-scale analysis of the cotton KCS gene family revealed a binary mode of action for gibberellin a regulated fiber growth. J Integr Plant Biol. 2016;58(6):577-89.

59. Qin Y-M, Zhu Y-X. How cotton fibers elongate: a tale of linear cell-growth mode. Curr Opin Plant Biol. 2011;14(1):106-11.

60. Basra AS, Malik CP. Development of the Cotton Fiber. In: Int Rev Cytol. Edited by Bourne GH, Danielli JF, Jeon KW, vol. 89: Academic Press; 1984: 65-113.

61. Stiff MR, Haigler $\mathrm{CH}$. Recent advances in cotton Fiber development. In: Flowering And Fruiting In Cotton. Edited by Oosterhuis DM, Cothren JT: The Cotton Foundation; 2012: 163-192.

62. Liu ZH, Zhu L, Shi HY, Chen Y, Zhang JM, Zheng Y, Li XB. Cotton GASL genes encoding putative gibberellin-regulated proteins are involved in response to GA signaling in fiber development. Mol Biol Rep. 2013;40(7): 4561-70.

63. Wang M, Wang $\mathrm{Q}$, Wang $\mathrm{B}$. Identification and characterization of microRNAs in Asiatic cotton (Gossypium arboreum L.). PLoS One. 2012;7(4):e33696.
64. Wang W, Liu D, Chen D, Cheng Y, Zhang X, Song L, Hu M, Dong J, Shen F. MicroRNA414C affects salt tolerance of cotton by regulating reactive oxygen species metabolism under salinity stress. RNA Biol. 2019;16(3):362-75.

65. Xie F, Sun G, Stiller JW, Zhang B. Genome-wide functional analysis of the cotton Transcriptome by creating an integrated EST database. PLoS One. 2011;6(11):e26980.

66. Wang W, Xia MX, Chen J, Yuan R, Deng FN, Shen FF. Gene expression characteristics and regulation mechanisms of superoxide dismutase and its physiological roles in plants under stress. Biochemistry Moscow. 2016;81(5): 465-80.

67. Zhu T, Liang C, Meng Z, Sun G, Meng Z, Guo S, Zhang R. CottonFGD: an integrated functional genomics database for cotton. BMC Plant Biol. 2017; 17(1):101.

68. Camacho C, Coulouris G, Avagyan V, Ma N, Papadopoulos J, Bealer K, Madden TL. BLAST+: architecture and applications. BMC Bioinformatics. 2009; 10:421

69. Finn RD, Coggill P, Eberhardt RY, Eddy SR, Mistry J, Mitchell AL, Potter SC, Punta M, Qureshi M, Sangrador-Vegas A, et al. The Pfam protein families database: towards a more sustainable future. Nucleic Acids Res. 2016;44(D1): D279-85.

70. Quevillon E, Silventoinen V, Pillai S, Harte N, Mulder N, Apweiler R, Lopez R. InterProScan: protein domains identifier. NAR. 2005;33(Web Server issue) W116-20.

71. Nordberg H, Cantor M, Dusheyko S, Hua S, Poliakov A, Shabalov I, Smirnova T, Grigoriev IV, Dubchak I. The genome portal of the Department of Energy Joint Genome Institute: 2014 updates. Nucleic Acids Res. 2014;42(Database issue):D26-31.

72. Yu J, Jung S, Cheng CH, Ficklin SP, Lee T, Zheng P, Jones D, Percy RG, Main D. CottonGen: a genomics, genetics and breeding database for cotton research. Nucleic Acids Res. 2014:42(Database issue):D1229-36.

73. Hu B, Jin J, Guo A-Y, Zhang H, Luo J, Gao G. GSDS 2.0: an upgraded gene feature visualization server. Bioinformatics. 2015;31(8):1296-7.

74. Yu CS, Chen YC, Lu CH, Hwang JK. Prediction of protein subcellular localization. Proteins. 2006;64(3):643-51.

75. Letunic I, Doerks T, Bork P. SMART: recent updates, new developments and status in 2015. Nucleic Acids Res. 2015:43(Database issue):D257-60.

76. Liu W, Xie Y, Ma J, Luo X, Nie P, Zuo Z, Lahrmann U, Zhao Q, Zheng Y, Zhao $Y$, et al. IBS: an illustrator for the presentation and visualization of biological sequences. Bioinformatics. 2015;31(20):3359-61.

77. Krzywinski M, Schein J, Birol I, Connors J, Gascoyne R, Horsman D, Jones SJ, Marra MA. Circos: an information aesthetic for comparative genomics. Genome Res. 2009;19(9):1639-45.

78. Lee $\mathrm{TH}$, Tang H, Wang X, Paterson AH. PGDD: a database of gene and genome duplication in plants. Nucleic Acids Res. 2013;41(Database issue): D1152-8.

79. Wang Y, Tang H, Debarry JD, Tan X, Li J, Wang X, Lee TH, Jin H, Marler B, Guo $\mathrm{H}$, et al. MCScanX: a toolkit for detection and evolutionary analysis of gene synteny and collinearity. Nucleic Acids Res. 2012;40(7):e49.

80. Thompson JD, Gibson TJ, Higgins DG. Multiple sequence alignment using ClustalW and ClustalX. Current Protocols in Bioinformatics 2002; Chapter 2: Unit 23.

81. Kumar S, Nei M, Dudley J, Tamura K. MEGA: a biologist-centric software for evolutionary analysis of DNA and protein sequences. Brief Bioinform. 2008; 9(4):299-306.

82. You Q, Xu W, Zhang K, Zhang L, Yi X, Yao D, Wang C, Zhang X, Zhao X, Provart NJ, et al. ccNET: database of co-expression networks with functional modules for diploid and polyploid Gossypium. Nucleic Acids Res. 2017;45(9): 5625-6.

83. Schmittgen TD, Livak KJ. Analyzing real-time PCR data by the comparative C(T) method. Nat Protoc. 2008;3(6):1101-8.

84. Singh VK, Mangalam AK, Dwivedi S, Naik S. Primer premier: program for design of degenerate primers from a protein sequence. BioTechniques. 1998;24(2):318-9.

85. Willems $E$, Leyns L, Vandesompele J. Standardization of real-time PCR gene expression data from independent biological replicates. Anal Biochem. 2008;379(1):127-9.

86. Tang Q-Y, Zhang C-X. Data processing system (DPS) software with experimental design, statistical analysis and data mining developed for use in entomological research. Insect Sci. 2013;20(2):254-60.

87. Lescot M, Dehais $P$, Thijs G, Marchal K, Moreau Y, Van de Peer Y, Rouze P Rombauts S. PlantCARE, a database of plant cis-acting regulatory elements 
and a portal to tools for in silico analysis of promoter sequences. Nucleic Acids Res. 2002;30(1):325-7.

88. Kozomara A, Griffiths-Jones S. miRBase: annotating high confidence microRNAs using deep sequencing data. Nucleic Acids Res. 2014; 42(Database issue):D68-73.

89. Zhang Z, Yu J, Li D, Zhang Z, Liu F, Zhou X, Wang T, Ling Y, Su Z. PMRD: plant microRNA database. Nucleic Acids Res. 2010;38(Database issue):D80613.

90. Dai X, Zhao PX. PsRNATarget: a plant small RNA target analysis server. Nucleic Acids Res. 2011;39(Web Server issue):W155-9.

\section{Publisher's Note}

Springer Nature remains neutral with regard to jurisdictional claims in published maps and institutional affiliations.

Ready to submit your research? Choose BMC and benefit from:

- fast, convenient online submission

- thorough peer review by experienced researchers in your field

- rapid publication on acceptance

- support for research data, including large and complex data types

- gold Open Access which fosters wider collaboration and increased citations

- maximum visibility for your research: over $100 \mathrm{M}$ website views per year

At BMC, research is always in progress.

Learn more biomedcentral.com/submissions 